\title{
Reduction of the Hodgkin-Huxley Equations to a Single-Variable Threshold Model
}

\author{
Werner M. Kistler \\ Wulfram Gerstner \\ J. Leo van Hemmen \\ Institut für Theoretische Physik, Physik-Department der TUMünchen, D-85747 Garch- \\ ing bei München, Germany
}

It is generally believed that a neuron is a threshold element that fires when some variable $u$ reaches a threshold. Here we pursue the question of whether this picture can be justified and study the four-dimensional neuron model of Hodgkin and Huxley as a concrete example. The model is approximated by a response kernel expansion in terms of a single variable, the membrane voltage. The first-order term is linear in the input and its kernel has the typical form of an elementary postsynaptic potential. Higher-order kernels take care of nonlinear interactions between input spikes. In contrast to the standard Volterra expansion, the kernels depend on the firing time of the most recent output spike. In particular, a zeroorder kernel that describes the shape of the spike and the typical afterpotential is included. Our model neuron fires if the membrane voltage, given by the truncated response kernel expansion, crosses a threshold. The threshold model is tested on a spike train generated by the HodgkinHuxley model with a stochastic input current. We find that the threshold model predicts 90 percent of the spikes correctly. Our results show that, to good approximation, the description of a neuron as a threshold element can indeed be justified.

\section{Introduction}

Neuronal activity is the result of a highly nonlinear dynamic process that was first described mathematically by Hodgkin and Huxley (1952), who proposed a set of four coupled differential equations. The mathematical analysis of these and other coupled nonlinear equations is known to be a hard problem, and an intuitive understanding of the dynamics is difficult to obtain. Hence, a simplified description of neuronal activity is highly desirable and has been attempted repeatedly, for example, by FitzHugh (1961); Rinzel (1985); Abbott and Kepler (1990); Av-Ron, Parnas, and Segel (1991); Kepler, Abbott, and Marder (1992); and Joeken and Schwegler (1995), to mention only a few. An example of a simple model of neuronal spike dynamics is the integrate-and-fire neuron, which dates back to Lapicque (1907) 
and has become quite popular in neural networks modeling (see, e.g., Usher, Schuster, and Niebur, 1993; Abbott \& van Vreeswijk, 1993; Tsodyks, Mitkov, \& Sompolinsky, 1993; Hopfield \& Herz, 1995).

In this article we aim at a generalized, and realistic, version of the integrate-and-fire model. In order to reduce the four nonlinear equations of Hodgkin and Huxley to a simplified model with a single, scalar variable $u$, we use the spike response method, which has been introduced previously (Gerstner, 1991, 1995; Gerstner \& van Hemmen, 1992). A spike is triggered at a time $t^{f}$ if the membrane potential $u$ approaches a threshold $\vartheta$ from below. For $t>t^{f}$ and $t^{f}$ being the most recent firing time, the membrane voltage $u$ in the reduced description is given by an expansion of the form,

$$
u(t)=\eta\left(t-t^{f}\right)+\int_{0}^{\infty} \mathrm{d} s \epsilon^{(1)}\left(t-t^{f} ; s\right) I(t-s)+\cdots
$$

The following spike is generated if $u(t)=\vartheta$ and $\frac{d}{d t} u(t)>0$, which defines the next firing time $t^{f}$, and so on. The kernels $\eta$ and $\epsilon$ have a direct neuronal interpretation. The first term $\eta\left(t-t^{f}\right)$ in the right-hand side of equation 1.1 describes the form of a spike and the typical afterpotential following it. The action potential is triggered at $t=t f$, it reaches its maximum after a time $\delta$, and it is followed by a period of hyperpolarization that extends over approximately $15 \mathrm{~ms}$ (see Figure 1a). From a different point of view, we can think of $\eta\left(t-t^{f}\right)$ as the neuron's response to the threshold crossing at $t^{f}$. Similarly, the kernel $\epsilon$ describes the response of the membrane potential to an input current $I$. More precisely, $\epsilon_{1}^{(1)}(\infty ; s)$ as a function of $s$ is the linear response to a small current pulse at time $s=0$ given that the neuron did not fire in the past. In biological terms, $\epsilon^{(1)}$ describes the form of the postsynaptic potential evoked by an input spike (see Figure 1b). Since the responsiveness of the membrane to input pulses is reduced during or shortly after an action potential, the form of the postsynaptic potential depends also on the time $t-t^{f}$ that has passed since the last output spike. Higher-order terms in equation 1.1 describe the nonlinear interaction between several input pulses.

We concentrate here on the four-dimensional set of equations proposed by Hodgkin and Huxley to describe the spike activity in the giant axon of the squid (Hodgkin \& Huxley, 1952). We consider it as a well-studied standard model of spike activity, even though it does not provide a correct description of spiking in cortical neurons of mammals. The methods introduced below are, however, more general and can also be applied to more detailed neuron models, which often involve tens or hundreds of variables (Yamada, Koch, \& Adams, 1989; Wilson, Bhalla, Uhley, \& Bower, 1989; Traub, Wong, Miles, \& Michelson, 1991; and Ekeberg et al., 1991). 


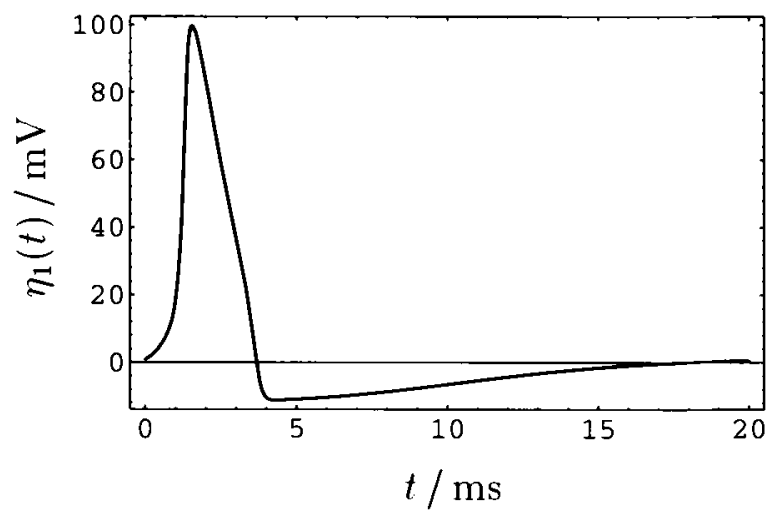

(a)

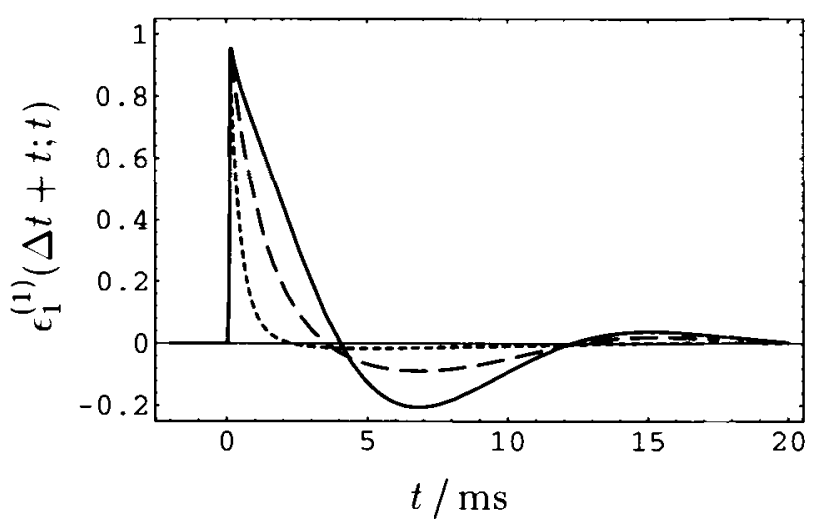

(b)

Figure 1: Kernels corresponding to the Hodgkin-Huxley equations and obtained by the methods described in this article. (a) The kernel $\eta_{1}$ describes the typical shape of a spike and the afterpotential. The spike has been triggered at time $t=0$. (b) The first-order kernel $\epsilon_{1}^{(1)}$ describes the form of the postsynaptic potential. The solid line in (b) shows the postsynaptic potential evoked by a short pulse of unit strength that has arrived at time $t=0$ while no spikes had been generated in the past. If there was an output spike $\Delta t=6.5 \mathrm{~ms}$ (dotted line) or $\Delta t=10.5 \mathrm{~ms}$ (dashed line) before the arrival of the input pulse, the response is significantly reduced. The solid line corresponds to $\Delta t=\infty$. 


\section{Theoretical Framework}

2.1 Standard Volterra Expansion. In order to approximate the membrane voltage $u$ of the Hodgkin-Huxley equations, we start with a Volterra expansion (Volterra, 1959),

$$
\begin{aligned}
u(t)= & \int_{0}^{\infty} \mathrm{d} s \epsilon_{0}^{(1)}(s) I(t-s) \\
& +\frac{1}{2 !} \int_{0}^{\infty} \mathrm{d} s \int_{0}^{\infty} \mathrm{d} s^{\prime} \epsilon_{0}^{(2)}\left(s, s^{\prime}\right) I(t-s) I\left(t-s^{\prime}\right)+\cdots
\end{aligned}
$$

The first-order term $\epsilon_{0}^{(1)}(s)$ describes the membrane's linear response to an external input current $I(t)$, the second-order term $\epsilon_{0}^{(2)}\left(s, s^{\prime}\right)$ takes into account nonlinear interactions between inputs at two different times, and the following terms take care of higher-order nonlinearities. We would like to stress that equation 2.2 is an ansatz whose usefulness we still have to verify. It is by no means evident that the series converges reasonably quickly.

2.2 Expansion During and After Spikes. When dealing with series expansions, we have to examine their convergence. As we will see, the expansion 2.1 converges as long as the input is so weak that no spikes are generated. If a spike is triggered at time $t^{f}$ by some strong input, the neuronal dynamics jumps onto a trajectory in phase space that is always (nearly) the same-the action potential. During the spike, the evolution is determined by nonlinear intrinsic processes and is hardly influenced by external input. We therefore replace equation 2.1 by

$$
\begin{aligned}
u(t)= & \eta_{1}\left(t-t^{f}\right)+\int_{0}^{\infty} \mathrm{d} s \epsilon_{1}^{(1)}\left(t-t^{f} ; s\right) I(t-s) \\
& +\frac{1}{2 !} \int_{0}^{\infty} \mathrm{d} s \int_{0}^{\infty} \mathrm{d} s^{\prime} \epsilon_{1}^{(2)}\left(t-t^{f} ; s, s^{\prime}\right) I(t-s) I\left(t-s^{\prime}\right)+\cdots
\end{aligned}
$$

Here $\eta_{1}\left(t-t^{f}\right)$ describes the evolution of the membrane potential during the spike and the relaxation process thereafter. In biological terms, $\eta_{1}\left(t-t^{f}\right)$ gives the form of a spike and the afterpotential. As in equation 2.1, the term $\epsilon_{1}^{(1)}$ describes the membrane's linear response to an input $I(t)$. Since inputs are much less effective during and shortly after a spike, the term $\epsilon_{1}^{(1)}$ depends on the time $t-t^{f}$ that has passed since the spike was triggered 
at $t^{f}$. The $\epsilon^{\prime} s$ are called response kernels. The formal arguments justifying equation 2.2 are discussed in the appendix.

The lower index of $\epsilon_{1}^{(1)}$ indicates that we take into account the effects of the most recent spike only. We can systematically increase the accuracy of our description by including more and more spikes that have occurred in the recent past. Taking into account the most recent $p$ spikes, we would have:

$$
\begin{aligned}
u(t)= & \eta_{p}\left(t-t_{1}^{f}, \ldots, t-t_{p}^{f}\right) \\
& +\int_{0}^{\infty} \mathrm{d} s \epsilon_{p}^{(1)}\left(t-t_{1}^{f}, \ldots, t-t_{p}^{f} ; s\right) I(t-s) \\
& +\frac{1}{2 !} \int_{0}^{\infty} \mathrm{d} s \int_{0}^{\infty} \mathrm{d} s^{\prime} \epsilon_{p}^{(2)}\left(t-t_{1}^{f}, \ldots, t-t_{p}^{f} ; s, s^{\prime}\right) I(t-s) I\left(t-s^{\prime}\right) \\
& +\cdots
\end{aligned}
$$

In general, $\eta_{p}\left(\sigma_{1}, \ldots, \sigma_{p}\right)$ is a complicated function of the $p$ arguments. Since we expect that adaptation is the dominant effect, we make the ansatz

$$
\eta_{p}\left(\sigma_{1}, \ldots, \sigma_{p}\right)=\sum_{k=1}^{p} \eta_{1}\left(\sigma_{k}\right) .
$$

Here $\eta_{1}(\sigma)$ describes a single spike and its afterpotential. A linear summation of the afterpotentials of several preceding spikes is often sufficient to describe the well-known adaptation effects of neuronal activity (Gerstner \& van Hemmen, 1992; Ekeberg et al., 1991; Kernell \& Sjöholm, 1973).

We will show that for the Hodgkin-Huxley model, excellent results are achieved with equation 2.2 , that is, with $p=1$. In other words, only the most recent output spike affects the dynamics. This is not too surprising since the Hodgkin-Huxley equations show no pronounced adaptation effects.

2.3 Relation to Integrate-and-Fire Models. In passing we note that the integrate-and-fire model is a special case of equation 2.3 with the response kernels (Gerstner, 1995)

$$
\begin{aligned}
\eta_{1}(\sigma) & =u_{0} \exp (-\sigma / \tau) \Theta(\sigma), \\
\epsilon_{1}^{(1)}(\sigma ; s) & =\tau^{-1} \exp (-s / \tau) \Theta(s) \Theta(\sigma-s),
\end{aligned}
$$

where $u_{0}$ is the voltage to which the system is reset after a spike, $\tau$ is the membrane time constant, and $\Theta($.$) is the Heaviside step function with \Theta(s)=1$ for $s>0$ and 0 otherwise. Since the standard integrate-and-fire model is linear except at firing, the determination of the kernels is simple. In particular, all terms beyond $\epsilon_{1}^{(1)}$ vanish. 
Equation 2.5 applies to the "one-step" integrate-and-fire model without synaptic or dendritic integration, but generalizations are straightforward. We mention three types of generalization:

1. The synaptic input current is not a $\delta$-pulse but is described by a broad pulse $\alpha(s)$ where $s=0$ is the time of presynaptic spike arrival. In this case, the response kernel $\epsilon_{1}^{(1)}$ has a finite rise time proportional to the width of the input pulse $\alpha$.

2. Dendritic integration can be incorporated by a broad input pulse $\tilde{\alpha}$. The effect is the same as in the first generalization.

3. We can include a time-dependent threshold $\vartheta_{0}+\vartheta(\sigma)$ where $\sigma$ is the time that has passed since the last output spike.

Points 1 and 3 are not relevant for a comparison with the Hodgkin-Huxley model, since the equations of Hodgkin and Huxley do not describe synaptic or dendritic transmission processes either. With respect to point 3 , it is easy to see that a time-dependent threshold is equivalent to changing the form of the $\eta_{1}$-kernel. The firing condition is

$$
\int_{0}^{\infty} \mathrm{d} s \epsilon_{1}^{(1)}(\sigma ; s) I(t-s)+\eta_{1}(\sigma)=\vartheta_{0}+\vartheta(\sigma) .
$$

Subtracting $\vartheta(\sigma)$ on both sides of the equation leads back to equation 1.1 with $\eta(\sigma)=\eta_{1}(\sigma)-\vartheta(\sigma)$.

In section 4.3, we test the performance of integrate-and-fire models with and without time-dependent threshold on a scenario with a fluctuating input current and compare the results with the Hodgkin-Huxley model.

2.4 Derivation of the Kernels. For complicated neuron models described by several nonlinear differential equations, the kernels can be derived systematically by the following procedure. In order to compute the response kernel $\epsilon_{0}^{(1)}$, we linearize the equations around the constant solution $u(t)=\bar{u}$ with zero input $I(t)=0$. The kernel $\epsilon_{0}^{(1)}$ is the voltage component of the Green's function of the linearized equation. Second and higher-order kernels can be obtained analytically by more involved methods. The mathematical details are in the Appendix.

We have explicitly calculated the kernels $\epsilon_{0}^{(1)}, \ldots, \epsilon_{0}^{(3)}$ for the HodgkinHuxley equations using a computer algebra system. Albeit the length of the expressions grows rather fast- the third-order kernel has about 400 simple terms - we found it more convenient, faster, and less consuming of computer memory to calculate the kernels analytically than using a numerical procedure.

It is instructive, however, to see how the kernels can be obtained numerically. To get the linear kernel, we solve the Hodgkin-Huxley equations 
numerically for an input current $I(t)=c i_{t_{0}}(t)$, where $i_{t_{0}}(t)$ is a short current pulse of unit strength at $t_{0}$, for example, $i_{t_{0}}(t)=\tau^{-1} \Theta\left(t-t_{0}+\tau / 2\right) \Theta(\tau / 2-$ $\left.t+t_{0}\right)$ with $\tau \ll 1 \mathrm{~ms}$. This is an approximate $\delta$-function. We denote the voltage component of the solution by $u\left[c i_{t_{0}}\right](t)$ and set $\epsilon_{0}^{(1)}(t)=\left\{u\left[c i_{t_{0}}\right](t-\right.$ $\left.\left.t_{0}\right)-\bar{u}\right\} / c$ in the limit $c \rightarrow 0$ and $\tau \rightarrow 0$, where $\bar{u}$ is the steady-state solution for zero input current. In a similar vein, we measure the voltage response to two current pulses so as to find $\epsilon_{0}^{(2)}$.

The improved kernel $\epsilon_{1}^{(1)}$ can only be found numerically. This is done in a similar way as above. We use an input current made up of two current pulses: $I(t)=c_{1} i_{t_{1}}(t)+c_{2} i_{t_{2}}(t)$. The first pulse at $t_{1}$ is strong enough to generate an output spike at $t^{f}$. The membrane potential after the spike serves as a reference trajectory $u\left[c_{1} i_{t_{1}}\right]$. Now we add the second pulse at time $t_{2}$ and consider the difference,

$$
\delta u=\lim _{\substack{c_{2} \rightarrow 0 \\ \mathrm{r} \rightarrow 0}} c_{2}^{-1}\left\{u\left[c_{1} i_{t_{1}}+c_{2} i_{t_{2}}\right]-u\left[c_{1} i_{t_{1}}\right]\right\}
$$

The difference $\delta u(t)$ gives the values of the linear response kernel $\epsilon_{1}^{(1)}(\sigma ; s)$ on a "diagonal line" with $(\sigma ; s)=\left(s+t_{2}-t^{f} ; s\right)$,

$$
\epsilon_{1}^{(1)}\left(s+t_{2}-t^{f} ; s\right)=\delta u\left(s+t_{2}\right)
$$

The procedure is repeated for variable offsets $t_{2}-t^{f}$ by changing the interval between the first and the second pulse. Since the effect of the spike at $t^{f}$ decreases for $t \gg t^{f}$, we have

$$
\lim _{\sigma \rightarrow \infty} \epsilon_{1}^{(1)}(\sigma ; s)=\epsilon_{0}^{(1)}(s)
$$

where $\epsilon_{0}^{(1)}(s)$ is the analytically derived kernel introduced above.

Finally, the kernel $\eta$ is determined by solving the Hodgkin-Huxley equations numerically with input $I(t)=c i_{t_{0}}(t)$. The amplitude $c$ has been chosen sufficiently large so as to evoke a spike. The exact value of $c$ is not important. We set $\eta_{1}\left(t-t^{f}\right)=\lim _{\tau \rightarrow 0} u\left[c i_{t_{0}}\right](t)-\bar{u}$ for $t>t_{0}$ where $t^{f}$ is the time when $u\left[c i_{t_{0}}\right](t)$ crosses a formal threshold $\vartheta$ and $\bar{u}$ is the constant solution with zero input. Once the amplitude $c$ is fixed, the kernel $\eta$ is uniquely determined as the form of an action potential with, apart from the triggering pulse, zero input current.

The only free parameter is the threshold $\vartheta$, which is to be found by an optimization procedure described below.

2.5 Comparison with Wiener Expansions. The approach indicated so far shows that all kernels can be found by a systematic and straightforward procedure. The kernels can be derived either analytically, as described in 
the appendix, or numerically by studying the system's response to input pulses on a small set of examples.

This is in contrast to the Wiener theory (Wiener, 1958; Palm \& Poggio, 1977), which analyzes the response of a system to gaussian white noise. Since Wiener's approach to the description of nonlinear systems is a stochastic one, the determination of the Wiener kernels requires large sets of input and output data (Palm \& Poggio, 1978; Korenberg, 1989). Exploiting the deterministic response of the system to well-designed inputs (short pulses) thus simplifies things significantly. The study of impulse response functions is a well-known approach to linear or weakly nonlinear systems. Here we have extended this approach to highly nonlinear systems under the proviso that the response kernels $\epsilon$ are (almost everywhere) continuous functions of their arguments.

It is important to keep in mind that Volterra and Wiener series cannot fully reproduce the threshold behavior of spike generation, even if higher-order terms are taken into account. The reason is that these expansions can only approximate mappings that are smooth, whereas the mapping from input current $l(t)$ to the time course of the membrane voltage has an apparent discontinuity at the spiking threshold (see Figure 2). Of course, this is not a discontinuity in the mathematical sense, but the output is very sensitive to small changes in the input current (Cole, Guttman, \& Bezanilla, 1970; Rinzel \& Ermentrout, 1989). We have to correct for this by adding the kernel $\eta$ each time the series expansion indicates that a spike will occur, say, by crossing an appropriate threshold value. In doing so, we no longer expand around the constant solution $u(t) \equiv 0$, but around $u(t)=\eta\left(t-t^{f}\right)$, the time course of a standard action potential.

The general framework outlined in this section will now be applied to the Hodgkin-Huxley equations.

\section{Application to Hodgkin-Huxley Equations}

Applying the theoretical analysis to the Hodgkin-Huxley equations, we begin by specifying the equations and then explain how the reduction to the spike response model is performed.

3.1 Hodgkin-Huxley Spike Trains. According to Hodgkin and Huxley (Hodgkin \& Huxley, 1952; Cronin, 1987), the voltage $v(t)$ across a small patch of axonal membran (e.g., close to the hillock) changes at a rate given by

$$
C \frac{d v}{d t}=I(t)-g_{N a} m^{3} h\left(v-v_{N a}\right)-g_{K} n^{4}\left(v-v_{K}\right)-g_{L}\left(v-v_{L}\right),
$$

where $I(t)$ is a time-dependent input current. The constants $v_{N a}, v_{K}$, and $v_{L}$ are the equilibrium potentials corresponding to sodium, potassium, and "leakage currents," and the $g$ 's are parameters of the respective ion conduc- 


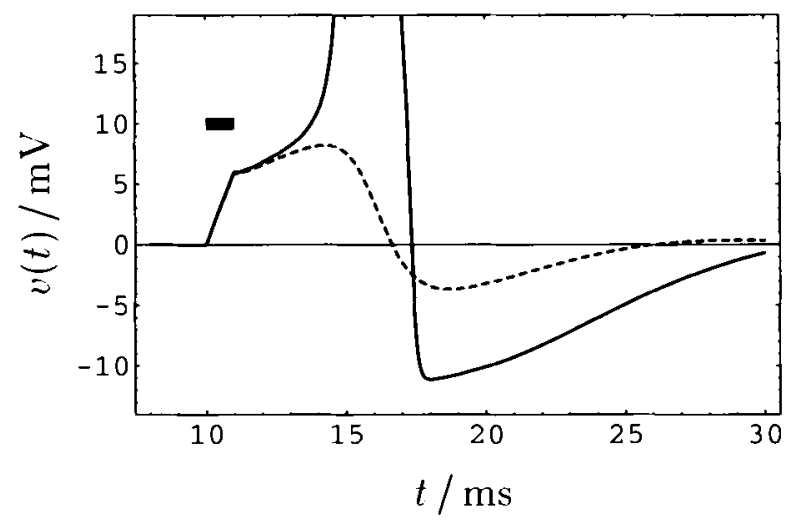

Figure 2: Threshold behavior of the Hodgkin-Huxley equations (see equations 3.1 and 3.2). We show the response of the Hodgkin-Huxley equations to a current pulse of $1 \mathrm{~ms}$ duration. A current amplitude of $7.0 \mu \mathrm{A} \mathrm{cm}^{-2}$ suffices to evoke an action potential (solid line; the maximum at $100 \mathrm{mV}$ is out of scale), whereas a slightly smaller pulse of $6.9 \mu \mathrm{A} \mathrm{cm}^{-2}$ fails to produce a spike (dashed line). The time course of the membrane voltage is completely different in both cases. Therefore, the mapping of the input current onto the time course of the membrane voltage is highly sensitive to changes in the input around the spiking threshold. The bar in the upper left indicates the duration of the input pulse.

tances. The variables $m, n$, and $h$ change according to a differential equation of the form

$$
\frac{d x}{d t}=\alpha_{x}(v)(1-x)-\beta_{x}(v) x
$$

with $x \in\{m, n, h\}$. The parameters are given in Table 1 .

For a biological neuron that is part of a larger network of neurons, the input current is due to spike input from many presynaptic neurons. Hence the input current is not constant but fluctuates. In our simulations, we therefore use an input current generated by the following procedure. Every $2 \mathrm{~ms}$, a random number is drawn from a gaussian distribution with zero mean and standard deviation $\sigma$. The discrete current values at intervals of $2 \mathrm{~ms}$ are linearly interpolated and define the input $I(t)$. This approach is intended to mimic the effect of spike input into the neuron. The procedure is somewhat arbitrary but easily implementable and leads to a spike train with a broad interval distribution and realistic firing rates depending on $\sigma$, as shown in Figure 3.

We emphasize that our single-variable model is intended to reproduce the firing times of the spikes generated by the Hodgkin-Huxley equations. 
Table 1: Parameters of the Hodgkin-Huxley Equations (Membrane Capacity, $C=1 \mu \mathrm{F} / \mathrm{cm}^{2}$ )

\begin{tabular}{ccc}
\hline$x$ & $v_{x}$ & $g_{x}$ \\
\hline $\mathrm{Na}$ & $115 \mathrm{mV}$ & $120 \mathrm{mS} / \mathrm{cm}^{2}$ \\
$K$ & $-12 \mathrm{mV}$ & $36 \mathrm{mS} / \mathrm{cm}^{2}$ \\
$L$ & $10.6 \mathrm{mV}$ & $0.3 \mathrm{mS} / \mathrm{cm}^{2}$ \\
\hline
\end{tabular}

\begin{tabular}{ccc}
\hline$x$ & $\alpha_{x}(v / \mathrm{mV})$ & $\beta_{x}(v / \mathrm{mV})$ \\
\hline$n$ & $(0.1-0.01 v) /[\exp (1-0.1 v)-1]$ & $0.125 \exp (-v / 80)$ \\
$m$ & $(2.5-0.1 v) /[\exp (2.5-0.1 v)-1]$ & $4 \exp (-v / 18)$ \\
$h$ & $0.07 \exp (-v / 20)$ & $1 /[\exp (3-0.1 v)+1]$ \\
\hline
\end{tabular}

This is a harder problem than fitting the gain function for constant input current.

3.2 Approximation by a Threshold Model. We want to reduce the four Hodgkin-Huxley equations (3.1 and 3.2) to the spike response model (see equation 2.2) in such a way that the model generates a spike train identical with or at least similar to the original one of Figure $3 a$. The reduction will involve four major steps:

1. Calculate the response functions $\epsilon_{0}^{(1)}, \epsilon_{0}^{(2)}$, and $\epsilon_{0}^{(3)}$.

2. Derive the kernel $\eta_{1}$.

3. Analyze the corrections to $\epsilon_{0}^{(1)}$ that are caused by the most recent output spike.

4. Determine a threshold criterion for firing.

Before starting, we note that for $I(t) \equiv \bar{I}$, the Hodgkin-Huxley equations allow a stationary solution with constant values $v(t)=\bar{v}, m(t)=\bar{m}, h(t)=\bar{h}$, and $n(t)=\bar{n}$. The constant solution is stable if $\bar{l}$ is smaller than a threshold value $\bar{I}_{\vartheta}=9.47 \mu \mathrm{A} / \mathrm{cm}^{2}$, which is determined by the parameter values given in Table 1.

3.2.1 Standard Volterra Expansion. The kernels $\epsilon_{0}^{(1)}\left(s_{1}\right)$ and $\epsilon_{0}^{(2)}\left(s_{1}, s_{2}\right)$ have been derived as indicated in the appendix and are shown in Figures $1 \mathrm{~b}$ (solid line) and 4. Figure 5 shows a small section of the spike train of Figure 3a with a single spike. Two intervals, one before (lower left) and a second during and immediately after the spike (lower right), are shown on an enlarged scale. Before the spike occurs, the numerical solution of the Hodgkin-Huxley equations (solid) is well approximated by the 


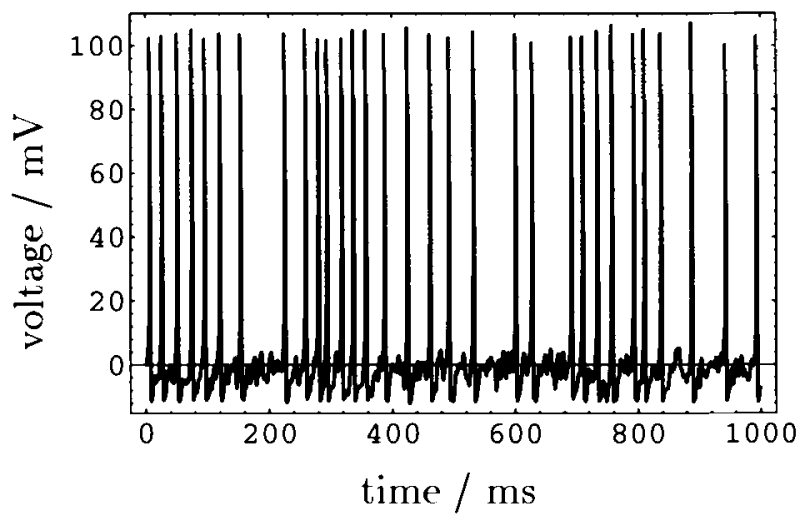

(a)

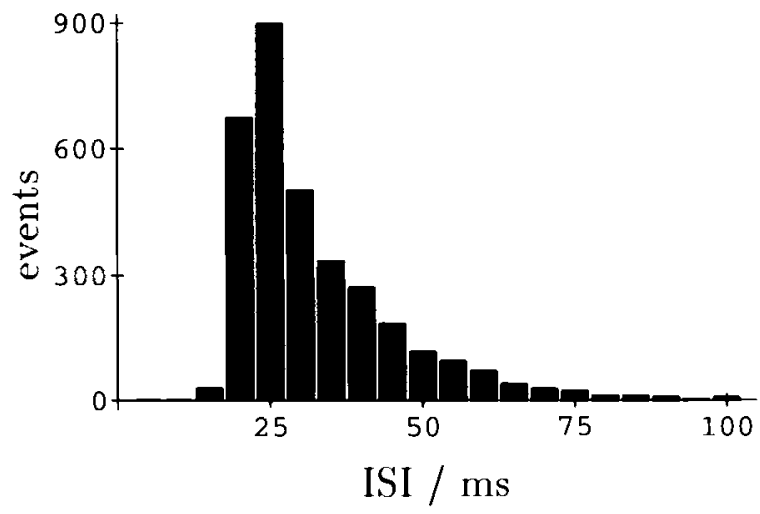

(b)

Figure 3: Hodgkin-Huxley model. (a) $1000 \mathrm{~ms}$ of a simulation of the HodgkinHuxley equations stimulated through a fluctuating input current $I(t)$ with zero mean and standard deviation $3 \mu \mathrm{A} / \mathrm{cm}^{2}$. Because of the fluctuating input, the spikes occur at stochastic intervals with a broad distribution. (b) A histogram of the interspike interval (ISI) sampled over a period of $100 \mathrm{~s}$.

first-order Volterra expansion $u(t)=\int d s \epsilon_{0}^{(1)}(s) I(t-s)$ (long dashed line) and even better by the second-order approximation (dotted line). During a spike, however, even an approximation to third order fails completely (see Figure 6).

The discrepancy during a spike may be surprising at first sight but is not completely unexpected. During action potential generation, the neuronal dynamics is mainly driven by internal processes of channel opening and closing, much more than by external input. Mathematically, the reason is 

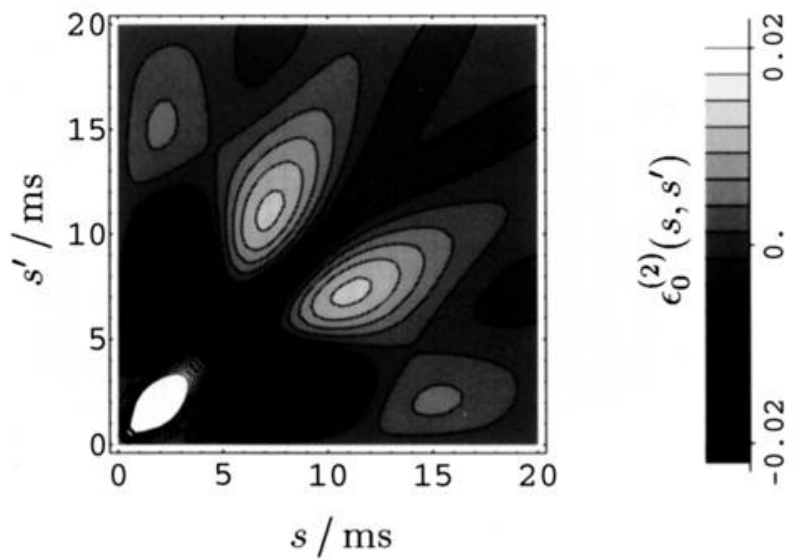

Figure 4: Second-order kernel for the Hodgkin-Huxley equations. The figure exhibits a contour plot for the second-order kernel $\epsilon_{0}^{(2)}\left(s, s^{\prime}\right)$. The kernel vanishes identically for negative arguments, as is required by causality.

that the series expansion is valid only as long as $I(t)$ is not too large; see the Appendix.

3.2.2 Expansion Including Action Potentials. In order to account for the remaining differences, we have to add explicitly the shape of the spike and the afterpotential by way of the kernel $\eta_{1}(s)$. The function $\eta_{1}(s)$ has been determined by the procedure explained in section 2 and is shown in Figure 1a. If we add $\eta_{1}\left(t-t^{f}\right)$ but continue to work with the standard kernels $\epsilon_{0}^{(1)}(s), \epsilon_{0}^{(2)}\left(s, s^{\prime}\right), \ldots$ we find large errors in an interval up to roughly $20 \mathrm{~ms}$ after a spike. This is exemplified in the lower right of Figure 5 where the longdashed line shows the approximation $u(t)=\eta_{1}\left(t-t^{f}\right)+\int d s \epsilon_{0}^{(1)}(s) I(t-s)$. From a biological point of view, this is easy to understand. The kernel $\epsilon_{0}^{(1)}(s)$ describes a postsynaptic potential in response to a standard input spike. Due to refractoriness, the responsiveness of the membrane is lower after a spike, and for this reason the postsynaptic potential looks different if an input pulse arrives shortly after an output spike. We then have to work with the improved kernels $\epsilon_{1}^{(1)}(\sigma ; s), \epsilon_{1}^{(2)}\left(\sigma ; s, s^{\prime}\right), \ldots$ introduced in equation 2.2. The dotted line in the lower-right graph of Figure 5 gives the approximation $u(t):=\eta_{1}\left(t-t^{f}\right)+\int d s \epsilon_{1}^{(1)}\left(t-t^{f} ; s\right) I(t-s)$, and we see that the fit is nearly perfect.

3.2.3 Threshold Criterion. The trigger time $t^{f}$ of an action potential is given by a simple threshold crossing process. Although the expansion in terms of the $\epsilon$ kernels will never give the perfect form of the spike, the 


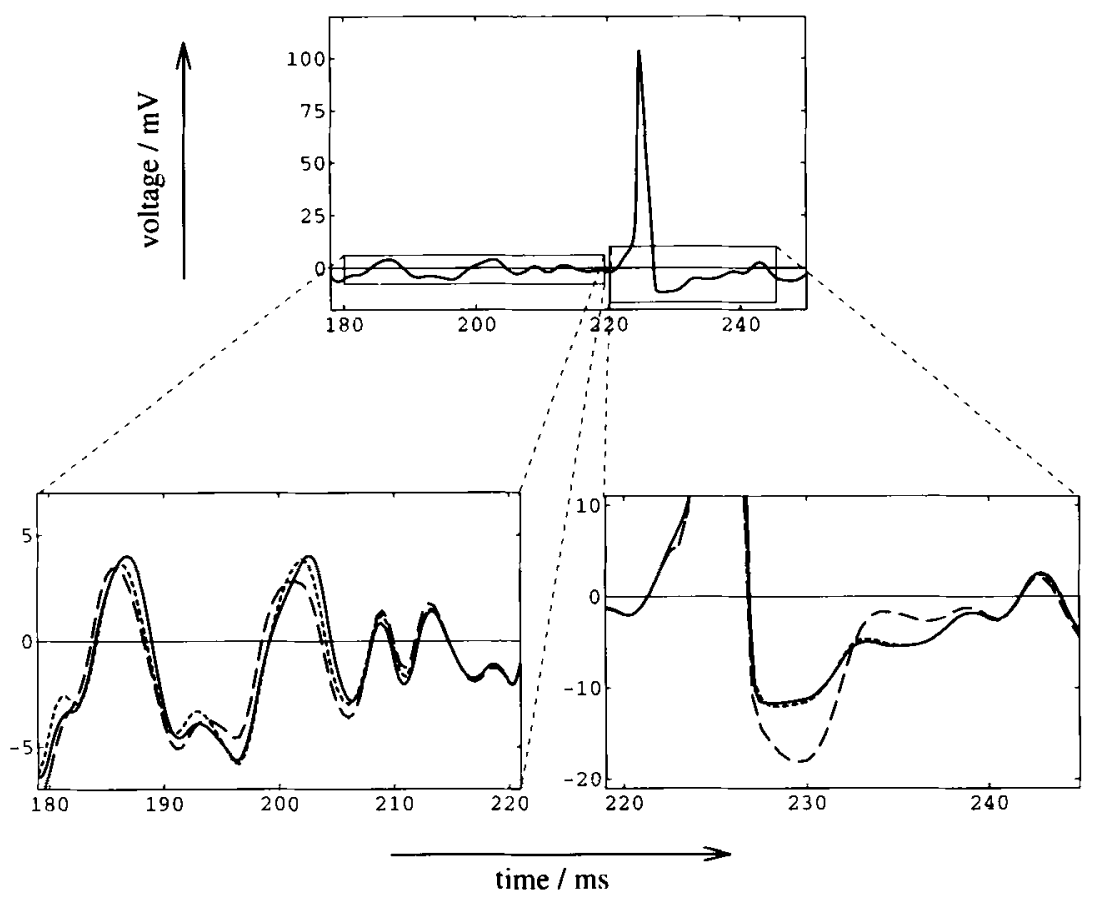

Figure 5: Systematic approximation of the Hodgkin-Huxley model by the spike response model (see equation 2.2). The upper graph shows a small section of the Hodgkin-Huxley spike train of Figure 3a. The lower left diagram shows the solution of the Hodgkin-Huxley equations (solid) and the first (dashed) and second (dotted) order approximation with the kernels $\epsilon_{0}^{(1)}$ and $\epsilon_{0}^{(2)}$ before the spike occurs on an enlarged scale. The lower right diagram illustrates the behavior of the membrane immediately after an action potential. The solution of the Hodgkin-Huxley equations (solid line) is approximated by $u(t)=\eta_{1}(t-$ $\left.t^{f}\right)+\int d s \epsilon_{0}^{(1)}(s) I(t-s)$, a dashed line. Using the improved kernel $\epsilon_{1}^{(1)}$ instead of $\epsilon_{0}^{(1)}$ results in a nearly perfect fit (dotted line).

truncated series expansion does exhibit rather significant peaks at positions where the Hodgkin-Huxley model produces spikes (see Figure 6). We therefore decided to apply a simple voltage threshold criterion instead of using some other derived quantity such as the derivative $\dot{v}$ or an effective input current (Koch, Bernander, \& Douglas, 1995). Whenever the membrane potential in terms of the expansion (see equation 2.3) reaches the threshold $\vartheta$ from below, we define a firing time $t^{f}$ and add a contribution $\eta\left(t-t^{f}\right)$. The threshold parameter $\vartheta$ is considered as a fit parameter and has to be optimized as described below. 


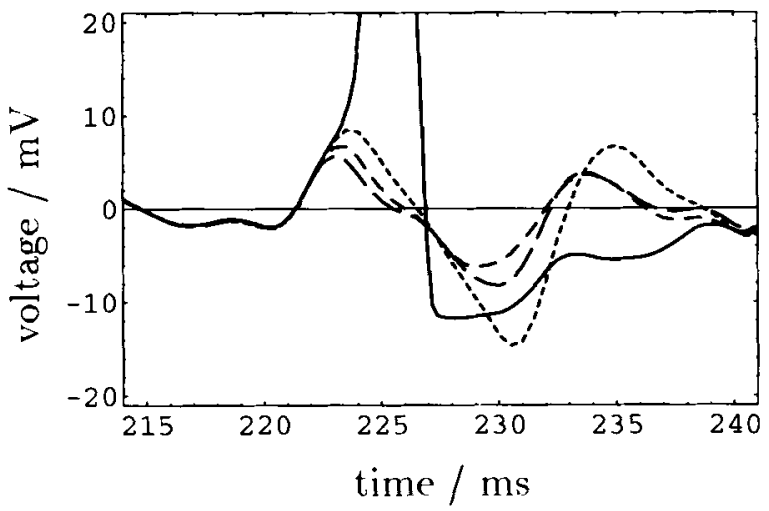

Figure 6: Approximation of the Hodgkin-Huxley model by a standard Volterra expansion (see equation 2.1) during a spike. Here we demonstrate the importance of the kernel $\eta_{1}$ as it appears in equation 2.2. First- (long dashed) and second-order approximation (dashed) using the kernels $\epsilon_{0}^{(1)}$ and $\epsilon_{0}^{(2)}$ produces a significant peak in the membrane potential where the Hodgkin-Huxley equations generate an action potential (solid line), but even the third-order approximation (dotted) fails to reproduce the spike with a peak of nearly $100 \mathrm{mV}$ (far out of scale). The remaining difference is taken care of by the kernel $\eta_{1}$.

\section{Simulation Results}

We have compared the full Hodgkin-Huxley model and the spike response model (see equation 2.2) in a simulation of spike activity over $100,000 \mathrm{~ms}$ (i.e., $100 \mathrm{~s}$ ). In so doing, we have accepted a spike of the spike response model to be coincident with the corresponding spike of the Hodgkin-Huxley model if it arrives within a temporal precision of $\pm 2 \mathrm{~ms}$.

4.1 Coincidence Measure. As a measure of the rate of coincidence of spikes generated by the Hodgkin-Huxley equations and another model such as equation 2.3 , we define an index $\Gamma$ that is the number of coincidences minus the number of coincidences by chance relative to the total number of spikes produced by the Hodgkin-Huxley and the other model. More precisely, $\Gamma$ is the fraction

$$
\Gamma=\frac{N_{\text {coinc }}-\left\langle N_{\text {coinc }}\right\rangle}{\frac{1}{2}\left(N_{\mathrm{HH}}+N_{\mathrm{SRM}}\right)} \mathcal{N}^{-1} .
$$

Here $N_{\text {coinc }}$ is the number of coincident spikes of Hodgkin-Huxley and the other model as counted in a single simulation run, $N_{\mathrm{HH}}$ the number of action potentials generated by the Hodgkin-Huxley equations, and $N_{\text {SRM }}$ the 
number of spikes produced by model, say, equation 2.2. The normalization factor $\mathcal{N}$ restricts $\Gamma$ to unity in the case of a perfect coincidence of the other model's spike train with the Hodgkin-Huxley one $\left(N_{\text {coinc }}=N_{\mathrm{SRM}}=N_{\mathrm{HH}}\right)$. Finally, $\left(N_{\text {coinc }}\right)$ is the average number of coincidences obtained if the spikes of our model were generated not systematically but randomly by a homogeneous Poisson process. Hence $\Gamma \approx 0$, if equation 2.2 would produce spikes randomly. The definition 4.1 is a modification of an idea of Joeken and Schwegler (1995).

In order to calculate $\left\langle N_{\text {coinc }}\right\rangle$, we perform a gedanken experiment. We are given the numbers $N_{\mathrm{HH}}$ and $N_{\mathrm{SRM}}$ and divide the total simulation time into $K$ bins of $4 \mathrm{~ms}$ length each. Due to refractoriness, each bin contains at most one Hodgkin-Huxley spike; we denote the number of these bins by $N_{\mathrm{HH}}$. So $\left(K-N_{\mathrm{HH}}\right)$ bins are empty. We now distribute $N_{\mathrm{SRM}}$ randomly generated spikes among the $K$ bins, each bin receiving at most one spike. A coincidence occurs each time a bin contains a Hodgkin-Huxley spike and a random spike. The probability of encountering $N_{\text {coinc }}$ coincidences is thus hypergeometrically distributed, that is, $p\left(N_{\text {coinc }}\right)=\left(\begin{array}{c}N_{\text {HH }} \\ N_{\text {ceinc }}\end{array}\right)\left(\begin{array}{c}K-N_{\text {HH }} \\ N_{\text {SRM }}-N_{\text {coinc }}\end{array}\right) /\left(\begin{array}{c}K \\ N_{\text {SRM }}\end{array}\right)$, with mean $\left\langle N_{\text {coinc }}\right\rangle=N_{\text {SRM }} N_{\mathrm{HH}} / K$. To see why, we use a simple analogy. Imagine an urn with $N_{\mathrm{HH}}$ black balls and $\left(K-N_{\mathrm{HH}}\right)$ white balls, and perform a random sampling of $N_{\text {SRM }}$ balls without replacement. The number of black balls drawn from the urn corresponds to the number of coincidences in the original problem. This setup is governed by the hypergeometric distribution (Prohorov \& Rozanov, 1969; Feller, 1970). In passing, we note that dropping the refractory side condition leads to a binomial distribution and, hence, to the same result for $\left\langle N_{\text {coinc }}\right\rangle$.

Using the correct normalization, $\mathcal{N}=1-N_{\mathrm{HH}} / K$, we thus obtain a suitable measure: $\Gamma$ has expectation zero for a purely random spike train, yields unity in case of a perfect coincidence of the spike response model's spike train with the Hodgkin-Huxley one, and is bounded by -1 from below. A negative $\Gamma$ hints at a negative correlation. Furthermore, $\Gamma$ is linear in the number of coincidences and monotonically decreasing with the number of erroneous spikes. That is, $\Gamma$ decreases with increasing $N_{\text {SRM }}$ while $N_{\text {coinc }}$ is kept fixed or with decreasing $N_{\text {coinc }}$ while $N_{\text {SRM }}$ is kept fixed.

While simulating the spike response model, we have found that due to the long-lasting afterpotential, a single misplaced spike causes subsequent spikes to occur with high probability at false times too. Since this is not a numerical artifact but a well-known and even experimentally observed biological effect (Mainen \& Sejnowski, 1995), we have adopted the following procedure in order to eliminate this type of problem from the statistics. Every time the spike response model fails to reproduce a spike of the HodgkinHuxley spike train, we note an error and add the kernel $\eta$ at the position where the original Hodgkin-Huxley spike occurred. Analogously, we count an error and omit the afterpotential if the spike response model produces a spike where no spike should occur. In case of a coincidence between the 
Hodgkin-Huxley spike and a spike of the spike response model, we take no action; we place the $\eta$ kernel at the position suggested by the threshold criterion. Without correction, $\Gamma$ would decrease by at most a few percentage points, and the standard deviation would increase by a factor of two.

4.2 Low Firing Rate. The results of the simulations for low mean firing rates are summarized in Table 2. Due to the large interspike intervals, effects from spikes in the past are rather weak. Hence we can ignore the dependence of the $\epsilon$ kernels on the former firing times and work with $\epsilon_{0}^{(1)}(s)$ instead of $\epsilon_{1}^{(1)}(\sigma ; s)$. We do, however, take into account the kernel $\eta_{1}(s)$. Higherorder kernels $\epsilon_{0}^{(2)}, \epsilon_{0}^{(3)}$ give a significant improvement over an approximation with $\epsilon_{0}^{(1)}$ only. This reflects the importance of the nonlinear interaction of input pulses. In a third-order approximation, the spike response model reproduces 85 percent of the Hodgkin-Huxley spikes correctly (see Table 2).

4.3 High Firing Rate. At higher firing rates, the influence of the most recent output spike is more important than the nonlinear interaction between input pulses. We therefore use the kernel $\epsilon_{1}^{(1)}$ but neglect higher-order terms with $\epsilon_{1}^{(2)}, \epsilon_{1}^{(3)}, \ldots$ in equation 2.2. Figure 7 gives the results of the simulations with different mean firing rates. As for Table 2, the threshold $\vartheta$ and the time $\delta$ between the formal threshold crossing time $t^{f}$ and the maximum of the action potential have been optimized in a separate run over $10 \mathrm{~s}$. For this optimization run we have used an input current with $\sigma=3 \mu \mathrm{A} / \mathrm{cm}^{2}$, corresponding to a mean firing rate of about $33 \mathrm{~Hz}$; the maximum mean firing rate is in the range of $70 \mathrm{~Hz}$.

For firing rates above $30 \mathrm{~Hz}$, the single-variable model reproduces about 90 percent of the Hodgkin-Huxley spikes correctly. This is quite remarkable since we have used only the first-order approximation,

$$
u(t)=\eta_{1}\left(t-t^{f}\right)+\int \mathrm{d} s \epsilon_{1}^{(1)}\left(t-t^{f} ; s\right) I(t-s),
$$

and neglected all higher-order terms. If we neglected the influence of the last spike, we would end up with a coincidence rate of only 73 percent, even in a second-order approximation using kernels $\epsilon_{0}^{(1)}$ and $\epsilon_{0}^{(2)}$.

Closer examination of the simulation results for various mean firing rates reveals a systematic deviation of the mean firing rate of the spike response model from the mean firing rate of the Hodgkin-Huxley equations. More precisely, the spike response model generates additional spikes where no spike should occur, and it misses fewer spikes if the standard deviation of the input current is increased - which is quite natural (Mainen \& Sejnowski, 1995).

We have performed the same test with two versions of traditional inte- 
Table 2: Simulation Results of the Spike Response Model Using the Volterra Kernels $\epsilon_{0}^{(1)}, \ldots, \epsilon_{0}^{(3)}$

\begin{tabular}{cccccccc}
\hline Order & $\vartheta / \mathrm{mV}$ & $\delta / \mathrm{ms}$ & $N_{\mathrm{SRM}}$ & $N_{\text {coinc }}$ & $N_{\text {wrong }}$ & $N_{\text {missed }}$ & $\Gamma$ \\
\hline First & 4.00 & 2.85 & $91 \pm 8$ & $71 \pm 7$ & $20 \pm 5$ & $16 \pm 3$ & $0.788 \pm 0.030$ \\
Second & 4.70 & 2.75 & $87 \pm 11$ & $74 \pm 8$ & $13 \pm 3$ & $13 \pm 2$ & $0.845 \pm 0.016$ \\
Third & 5.80 & 2.50 & $88 \pm 10$ & $76 \pm 8$ & $13 \pm 3$ & $11 \pm 3$ & $0.858 \pm 0.019$ \\
\hline
\end{tabular}

Note: The table gives the number of spikes produced by the spike response model $\left(N_{\mathrm{SRM}}\right)$, the number of coincidences ( $\left.N_{\text {coinc }}\right)$, the number of spikes produced by the spike response model where no spike should occur ( $N_{\text {wrong }}$ ), and the number of missing spikes ( $\left.N_{\text {missed }}\right)$. The numbers give the average and standard deviation after 10 runs with different realizations of the input current and a duration of 10 s per run. The numbers should be compared with the number of spikes in the Hodgkin-Huxley model, $N_{\mathrm{HH}}=87 \pm 8$. The coincidence rate $\Gamma$ has been defined by equation 4.1. For random gambling we would obtain $\Gamma \approx 0$. The parameters $\vartheta$ (threshold) and $\delta$ (time from threshold crossing to maximum of the action potential) have been determined by optimizing the results in a separate run over $10 \mathrm{~s}$.

grate-and-fire models: a simple integrate-and-fire model with reset potential $u_{0}$ and time constant $\tau$, and an integrate-and-fire model with timedependent threshold derived from the $\eta$-kernel found by the methods described in section 2.4- $\vartheta(\sigma)=-\eta_{1}(\sigma)$ for $\sigma>5 \mathrm{~ms}$ and $\vartheta(\sigma)=\infty$ for $\sigma<5 \mathrm{~ms}$. The results are summarized in Figure 8. We have optimized the parameters $\tau, \vartheta_{0}$, and $u_{0}$ with a fluctuating input current as already explained and found optimal performance for a time constant of $\tau=1 \mathrm{~ms}$. This surprisingly short time constant should be compared to the time constants of the $\epsilon_{1}^{(1)}$ kernel. Indeed, if the time since the last spike is shorter than about $10 \mathrm{~ms}$ (see Figure 1b), the $\epsilon_{1}^{(1)}$ kernel approaches zero within a few milliseconds. The optimized reset value was $u_{0}=-3 \mathrm{mV}$ and the threshold $\vartheta_{0}=3.05 \mathrm{mV}$ respectively $\vartheta_{0}=2.35 \mathrm{mV}$ for the model with respectively without time-dependent threshold.

4.4 Response to Current Step Functions. Constant input currents have been a paradigm to neuron models ever since Hodgkin and Huxley (1952), although a constant current is very far from reality and one may wonder whether it offers a sensible criterion for the behavior of neurons producing spikes and, thus, responding to fluctuating input currents. Nevertheless, we want to study the response of our model to current steps and compare it with that of the original Hodgkin-Huxley model.

As a consequence of the oscillatory behavior of the first-order kernel $\epsilon_{0}^{(1)}$ (see Figure 1b), our model exhibits a phenomenon known as inhibitory re- 


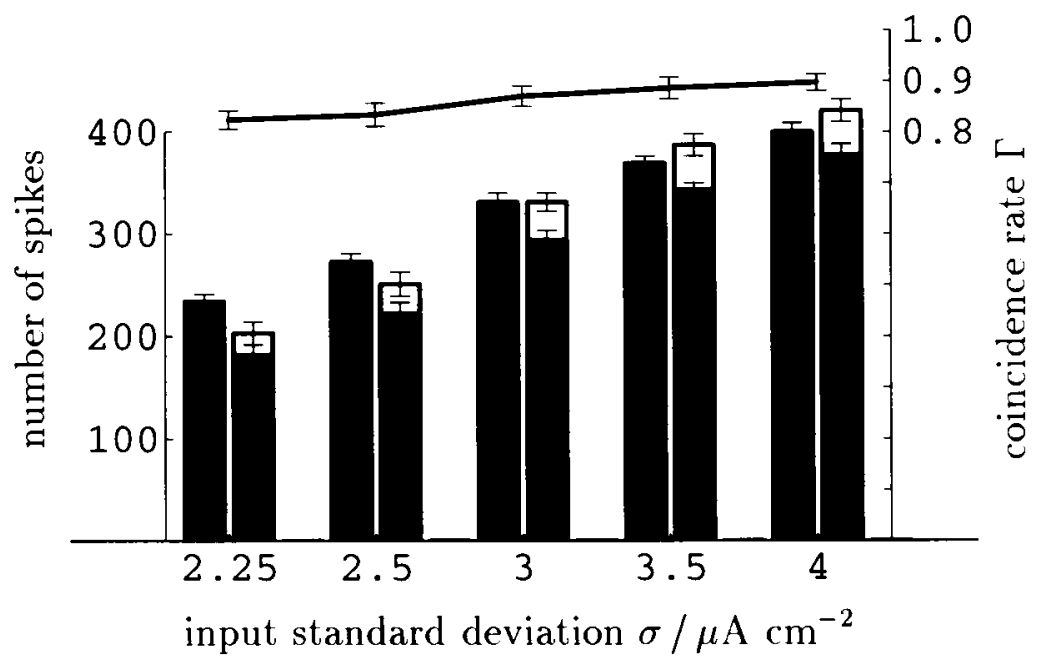

Figure 7: Simulation results for different mean firing rates using the first-order improved kernel $\epsilon_{1}^{(1)}$ only. The black bars indicate the number of spikes (left axis) produced by the Hodgkin-Huxley model; the neighboring bars correspond to the number of spikes of the spike response model. The gray shading reflects the coincident spikes. All numbers are averages of 10 runs over $10 \mathrm{~s}$ with different realizations of the input current. The mean firing rate varies between 23 and $40 \mathrm{~Hz}$. The error bars are the corresponding standard deviations. The line in the upper part of the diagram indicates the coincidence rate $\Gamma$ (right axis) as defined by equation 4.1 . The parameters $\vartheta=4.7 \mathrm{mV}$ and $\delta=2.15 \mathrm{~ms}$ have been optimized in a separate run with an input current with standard deviation $\sigma=3 \mu \mathrm{A} \mathrm{cm}^{-2}$.

bound. Suppose we have a constant inhibitory (i.e., negative) input current for time $t<0$, which is turned off suddenly at $t=0$. We can easily calculate the membrane potential from equation 1.1 if we assume that there are no spikes in the past. The resulting voltage trace exhibits a significant oscillation (see Figure 9). If the current step is large enough, the positive overshooting triggers a single spike after the release of the inhibition. Since equation 1.1 is linear in the input, the amplitude of the oscillatory response of the membrane potential in Figure 9 is proportional to the height of the current step but independent of the absolute current values before or after the step. This is in contrast to the Hodgkin-Huxley equations, where amplitude and frequency of the oscillations depend on both step height and absolute current values (Mauro, Conti, Dodge, \& Schor, 1970).

The limitations of a voltage threshold for spike prediction can be investi- 


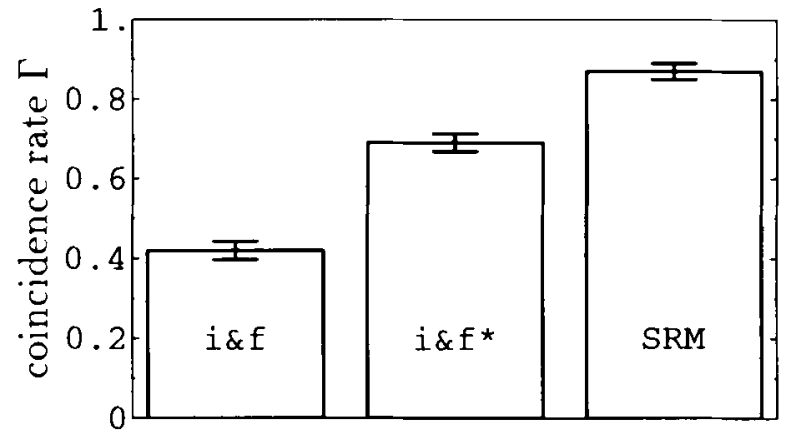

Figure 8: Comparison of various threshold models. The diagram shows the coincidence rate $\Gamma$ defined in section 4.1 for a simple integrate-and-fire model with time constant $\tau=1 \mathrm{~ms}$ and reset potential $u_{0}=-3 \mathrm{mV}$ (i\&f), an integrateand-fire model with time-dependent threshold (i\& $\left.\mathrm{f}^{*}\right)$, and the spike response model (SRM). The error bars indicate the standard deviation of 10 simulations over $10 \mathrm{~s}$ each with a fluctuating input current with $\sigma=3 \mu \mathrm{A} \mathrm{cm}^{-2}$.

gated by systematically studying the response of the model to current steps (see Figure 10). For $t<0$ we apply a constant current with amplitude $I_{1}$. At $t=0$ we switch to a stronger input $I_{2}>I_{1}$. Depending on the values of the final input current $I_{2}$ and the step height $\Delta I=I_{2}-I_{1}$ we observe three different modes of behavior in the original Hodgkin-Huxley model, as is well known (Cronin 1987). For small steps and low final input currents, the membrane potential exhibits a transient oscillation but no spikes (inactive phase I). Larger steps can induce a single spike immediately after the step (single-spike phase S). Increasing the final input current further leads to repetitive firing (R). Note that repetitive firing is possible for $I>6 \mu \mathrm{A} \mathrm{cm}^{-2}$, but firing must be triggered by a sufficiently large current step $\Delta I$. Only for currents larger than $9.47 \mu \mathrm{A} / \mathrm{cm}^{2}$ is there autonomous firing independent of the current step. We conclude from the step response behavior that there are two different threshold paradigms: a single-spike threshold (dashed line in Figure 10) and a threshold for repetitive firing (solid line).

We want to compare these results with the behavior of our threshold model. The same set of step response simulations has been performed with the spike response model. Comparing the threshold lines in the $\left(I_{2}, \Delta I\right)$ diagram for the Hodgkin-Huxley model and the spike response model, we can state a qualitative correspondence. The spike response model exhibits the same three modes of response as the Hodgkin-Huxley equations. Let us have a closer look at the two thresholds: the single-spike threshold and the repetitive firing threshold. The slope of the single-spike threshold (dashed 


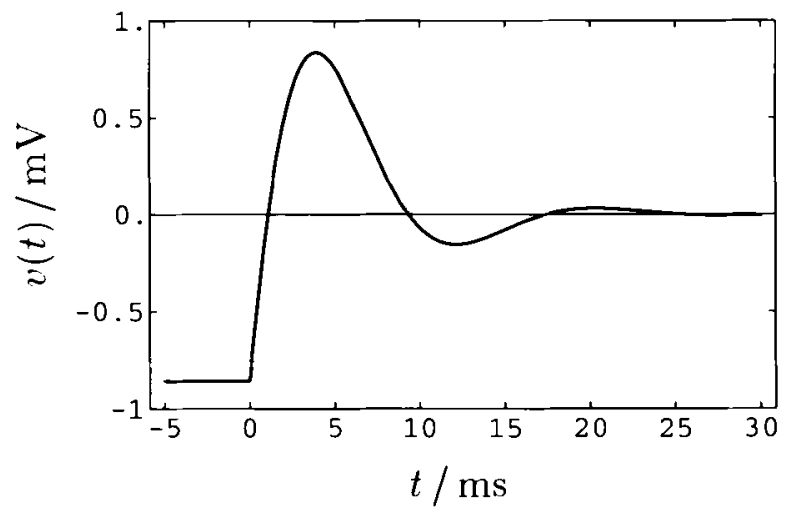

Figure 9: Response of the spike response model to a current step. An inhibitory input current that is suddenly turned off produces a characteristic oscillation of the membrane potential. The overshooting membrane potential can trigger an action potential immediately after the inhibition is turned off. The amplitude of the oscillation is proportional to the height of the current step. Here, the current is switched from $-1 \mu \mathrm{A} \mathrm{cm} \mathrm{cm}^{-2}$ to 0 at time $t=0$.

line in Figure 10) is far from perfect if we compare the lower and the upper parts of the figure. The slope is determined by the mean $\int \mathrm{d} s \epsilon_{0}^{(1)}(s)$ of the linear response kernel $\epsilon_{0}^{(1)}$ and is therefore fixed as long as we stick to the membrane voltage as the relevant variable used in applying the threshold criterion.

We now turn to the threshold of repetitive firing. The position of the vertical branch of the repetitive-firing threshold (solid line in Figure 10) is shifted to lower current values as compared to the Hodgkin-Huxley model. Consequently, the gain function of the spike response model is shifted to lower current values too (see Figure 11). The repetitive-firing threshold is directly related to the (free) threshold parameter $\vartheta$ and can be moved to larger current values by increasing $\vartheta$. Using $\vartheta=9 \mathrm{mV}$ instead of $\vartheta=$ $4.7 \mathrm{mV}$ results in a reasonable fit of the Hodgkin-Huxley gain function (see Figure 11). However, a shift of $\vartheta$ would also shift the single-spike threshold of Figure 10 to larger current values, and the triggering of single spikes at low currents would be made practically impossible. The value for the threshold parameter $\vartheta=4.7 \mathrm{mV}$ found by optimization with a fluctuating input as described in section 4.3 is therefore a compromise.

It follows from Figure 10 that there is not a strict voltage threshold for spiking in the Hodgkin-Huxley model. Nevertheless, our model qualitatively exhibits all relevant phases, and, with the fluctuating input scenario, there is even a fine quantitative agreement. 

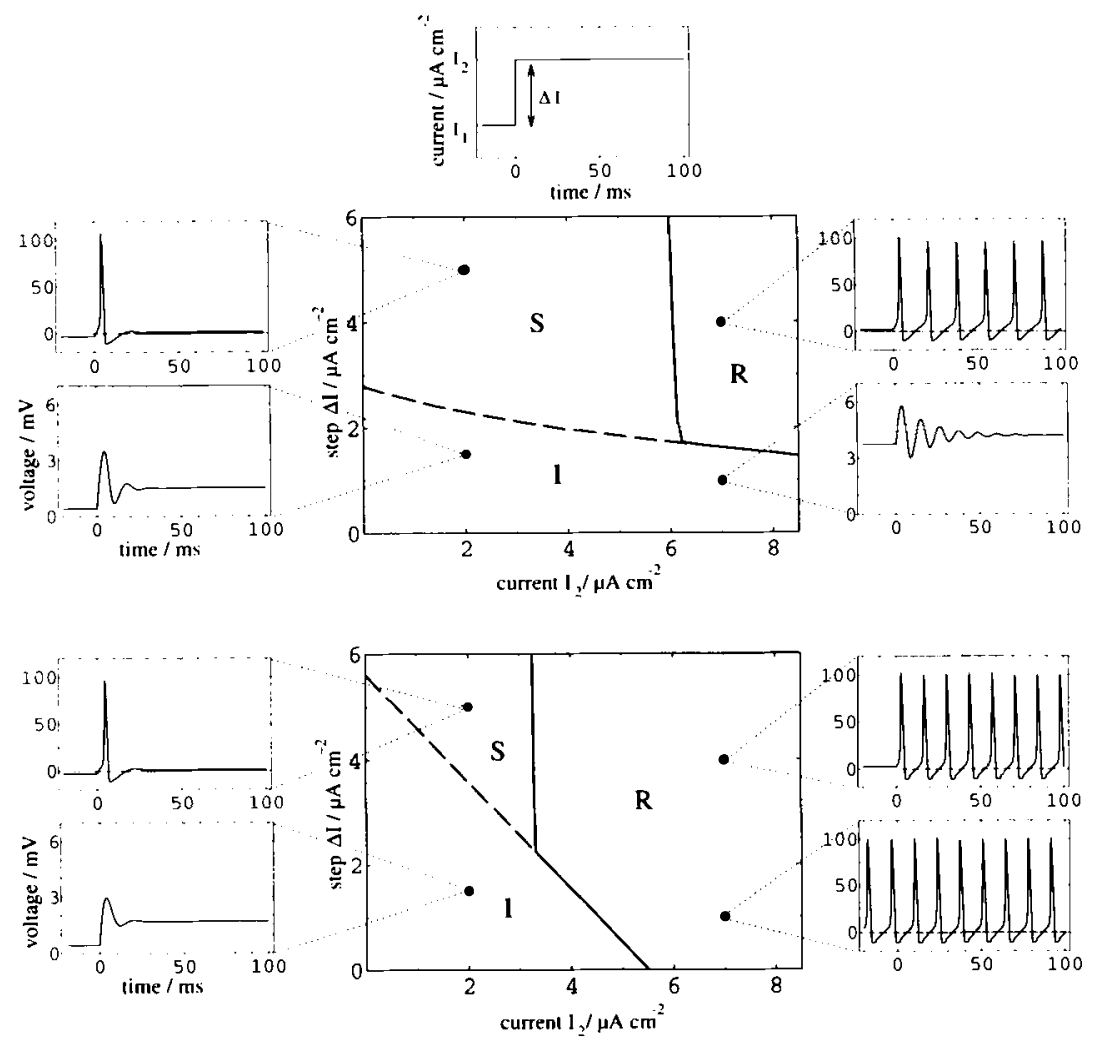

Figure 10: Comparison of the response to current steps of the Hodgkin-Huxley equations (upper graph) and the spike response model (lower graph). At time $t=0$ the input current is switched from $I_{1}$ to $I_{2}$, and the behavior of the models is studied in dependence on the final current $I_{2}$ and the step height $\Delta I=I_{2}-$ $I_{1}$. The figures in the center indicate in the manner of a phase diagram three different regimes: (I) the neuron remains inactive; $(S)$ a single spike is triggered; (R) repetitive firing is induced. We have chosen four representative pairs of values of $I_{2}$ and $\Delta I$ (marked by dots in the phase diagram) and plotted the corresponding time course of the membrane potential on the left and the right of the main diagram so that the responses of Hodgkin-Huxley model and spike response model can be compared. The parameters for the spike response model are the same as those detailed in the caption to Figure 7. 


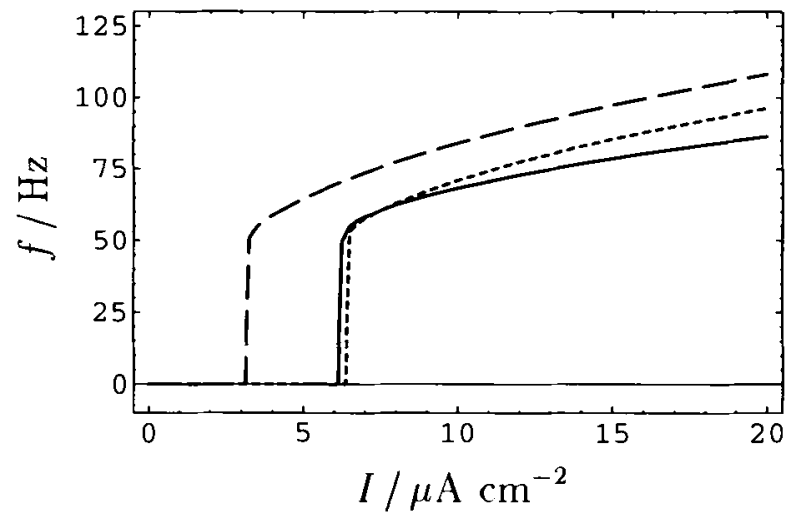

Figure 11: Comparison of the gain function (output firing rate for constant input current $I$ as a function of $I$ ) of the spike response and the Hodgkin-Huxley model. The threshold parameter $\vartheta=4.7 \mathrm{mV}$ found by the optimization procedure described in section 4.3 gives a gain function of the spike response model (dashed line) that is shifted toward lower current values as compared to the gain function of the Hodgkin-Huxley model (solid line). Increasing the threshold parameter to $\vartheta=9 \mathrm{mV}$ results in a reasonable fit (dotted line).

\section{Discussion}

In contrast to the complicated dynamics of the Hodgkin-Huxley equations, the spike response model in equation 2.2 has a straightforward and intuitively appealing interpretation. Furthermore, the transparent structure of the model has a great number of advantages. The dynamical behavior of a single neuron can be discussed in simple but biological terms of threshold, postsynaptic potential, refractoriness, and afterpotential.

As a first example, we discuss the refractory period. Refractoriness of the Hodgkin-Huxley model leads to two distinct effects. On the one hand, we have shunting effects-a reduced responsiveness of the membrane to input spikes during and immediately after the spike-that is related to absolute refractoriness and is expressed in our model by the first argument of $\epsilon_{1}^{(1)}$. In addition, the kernel $\eta_{1}$ induces an afterpotential corresponding to a relative refractory period. In the Hodgkin-Huxley model, the afterpotential corresponds with hyperpolarization and lasts for about $15 \mathrm{~ms}$, followed by a rather small depolarization. A different form of the afterpotential with a significant depolarizing phase would lead to intrinsic bursting (Gerstner \& van Hemmen, 1992). So the neuronal behavior is easily taken care of by the spike response model.

As a second example, let us focus on the temperature dependence of the various kernels. Temperature can be included in the Hodgkin-Huxley equa- 
tions by multiplying the right-hand side of equation 3.2 by a temperaturedependent factor $\zeta$ (Cronin, 1987),

$$
\zeta=\exp [\ln (3.0)(T-6.3) / 10.0]
$$

with the temperature $T$ in degrees Celsius. Equation 3.1 remains unmodified. In Figure 12 we have plotted the resulting $\epsilon_{0}^{(1)}$ and $\eta_{1}$ kernels for different temperatures. Although the temperature correction affects only the equations for $m, h$, and $n$, the overall effect can be approximated by a time rescaling, since the form of both kernels is not modified but stretched in time with decreasing temperature.

Apart from the transparent structure, the single-variable model is open to a mathematical analysis that would be inconceivable for the full HodgkinHuxley equations. Most important, the collective behavior of a large network of neurons can now be predicted if the form of the kernels $\eta$ and $\epsilon^{(1)}$ is known (Gerstner \& van Hemmen, 1993; Gerstner, 1995; Gerstner, van Hemmen, \& Cowan, 1996). In particular, the existence and stability of collective oscillations can be studied by a simple graphical construction using the kernels $\eta$ and $\epsilon^{(1)}$. It can be shown that a collective oscillation is stable if the sum of the postsynaptic potentials is increasing at the moment of firing. Otherwise it is unstable (Gerstner et al., 1996). Furthermore, it can be shown that in a fully connected and homogeneous network of spike response neurons with a potential described by equation 1.1, the state of asynchronous firing is almost always unstable (Gerstner, 1995). Also in simulations of a network of Hodgkin-Huxley neurons, a spontaneous breakup of the asynchronous firing state and a small oscillatory component in the mean firing activity have been observed (Wang, personal communication, 1995).

In summary, we have used the Hodgkin-Huxley equations as a wellstudied reference model of spike dynamics and shown that it can indeed be reduced to a threshold model. Our methods are more general and can also be applied to more elaborate models that involve many ion currents and a complicated spatial structure. Furthermore, an analytic evaluation of some of the kernels (see the appendix for mathematical details) is possible. We have also presented a numerical algorithm to determine the response kernels that, in contrast to the Wiener method, can be determined quickly and easily. In fact, the spike response method we have used in our analysis can be seen as a systematic approach to a reduction of a complex system to a threshold model.

\section{Appendix}

In this appendix we treat Volterra's theory (Volterra, 1959) in the context of inhomogeneous differential equations, indicate under what conditions and how the kernels of the Volterra series can be computed analytically, and isolate the mathematical essentials of our own approach. 


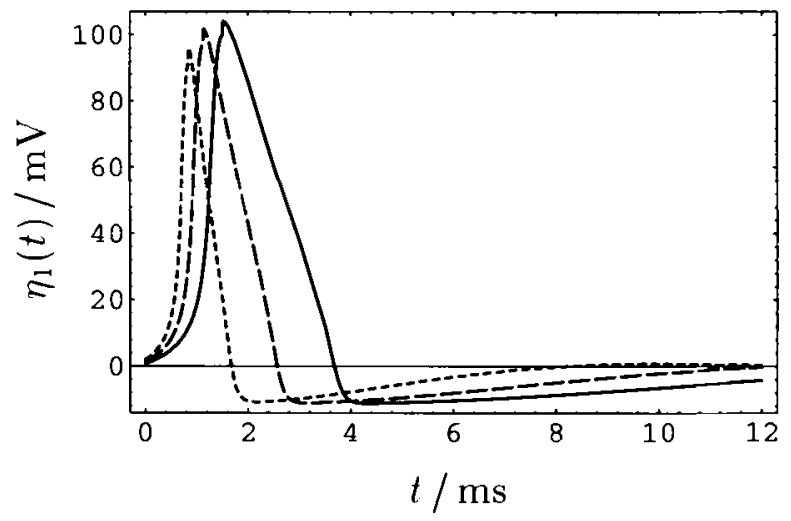

(a)

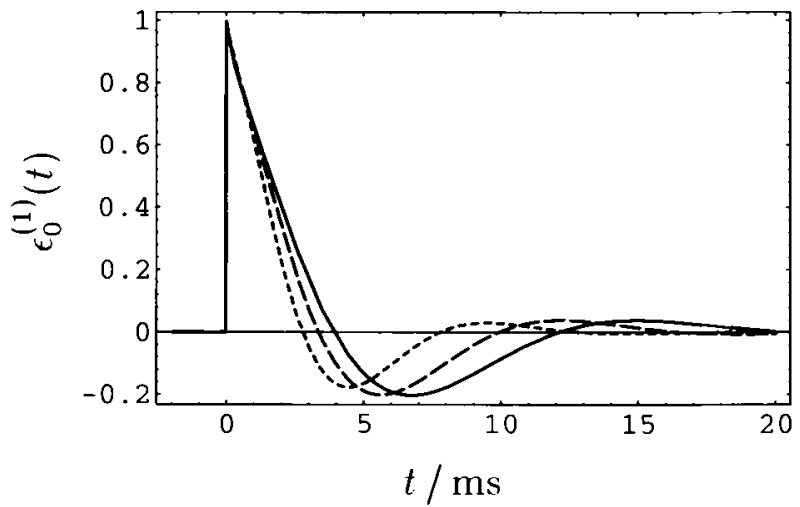

(b)

Figure 12: Temperature dependence of the kernels corresponding to the Hodgkin-Huxley equations. Both $\eta_{1}$ kernel (a) and $\epsilon_{0}^{(1)}$ kernel (b) show apart from a stretching in time no significant change in form if temperature is decreased from 15.0 (dotted line) and 10.0 (dashed line) to 6.3 (solid line) degrees Celsius.

A.1 Volterra Series. We start by studying the ordinary differential equation

$$
\dot{x}-f(x)=j
$$

where $j$ is a given function of time and $\dot{x}$ denotes differentiation of $x(t)$ with respect to $t$. We require that for $j \equiv 0, x=0$ should be an attractive fixed point with a sufficiently large basin of attraction so that the system returns 
to equilibrium if the perturbation $j(t)$ is turned off. Furthermore, we require $f$ and $j$ to be continuous and bounded and $f$ to fulfill a Lipschitz condition. Finally, $j \in \mathcal{L}^{2}(\mathbb{R})$ should have compact support so that $x \in \mathcal{L}^{2}(\mathbb{R})$.

Under these premises there exists a unique solution $x$, with $x(-\infty)=0$ for each input $j \in \mathcal{A} \subset \mathcal{L}^{2}(\mathbb{R})$. Hence, the time course of the solution $x$ is a function ${ }^{1}$ of the time course of the perturbation $j$, formally,

$$
\begin{aligned}
F: \mathcal{A} \subset \mathcal{L}^{2}(\mathbb{R}) & \rightarrow \mathcal{L}^{2}(\mathbb{R}), \\
j & \mapsto F[j]=x \quad \text { with } \quad \dot{x}(t)-f(x(t))=j(t), \quad \forall t \in \mathbb{R} .(A)
\end{aligned}
$$

Let us suppose that the function $F$ is analytic in a neighborhood of the constant solution $x \equiv 0$. For a precise definition of the notion of analyticity and conditions under which it holds, we refer to Thomas (1996). In case of analyticity there is a Taylor series (Dieudonné, 1968; Dunford \& Schwartz, 1958; Kolmogorov \& Fomin, 1975; Hille \& Phillips, 1957) for $F$,

$$
F[j]=0+F^{\prime}[0][j]+\frac{1}{2 !} F^{\prime \prime}[0][j, j]+\cdots
$$

This series is convergent with respect to the $\|\cdot\|_{2}$-norm, that is, $F[j](t)=x(t)$ for almost all $t \in \mathbb{R}$. Each derivative $F^{(n)}[0]$ in equation A.3 is a continuous $n$ linear mapping from $\left(\mathcal{L}^{2}(\mathbb{R})\right)^{n}$ to $\mathcal{L}^{2}(\mathbb{R})$. Hence, $F^{(n)}[0][].(t):\left(\mathcal{L}^{2}(\mathbb{R})\right)^{n} \rightarrow \mathbb{R}$ is a continuous $n$-linear functional for almost all (fixed) $t \in \mathbb{R}$. We know that every such functional has a Volterra-like integral representation (Volterra, 1959; Palm \& Poggio, 1977; Palm, 1978),

$$
F^{(n)}[0][j](t)=\int \mathrm{d} t_{1} \ldots \int \mathrm{d} t_{n} \epsilon_{t}^{(n)}\left(t_{1}, \ldots, t_{n}\right) j\left(t-t_{1}\right) \ldots j\left(t-t_{n}\right)
$$

In the most general case the kernels $\epsilon_{t}^{(n)}$ are distributions. We will see, however, that the kernels describing equation A.2 are (almost everywhere) continuous functions from $\mathcal{L}^{2}\left(\mathbb{R}^{n}\right)$.

Combining equations A.3 and A.4 yields the Volterra series of $F$,

$$
\begin{aligned}
x(t)= & \int \mathrm{d} t_{1} \epsilon^{(1)}\left(t_{1}\right) j\left(t-t_{1}\right) \\
& +\frac{1}{2 !} \int \mathrm{d} t_{1} \int \mathrm{d} t_{2} \epsilon^{(2)}\left(t_{1}, t_{2}\right) j\left(t-t_{1}\right) j\left(t-t_{2}\right)+\cdots
\end{aligned}
$$

${ }^{1}$ The bracket convention is such that (..) denotes the dependence on a real or complex scalar, and [..] denotes the dependence on a function. 


$$
\begin{aligned}
& +\frac{1}{n !} \int \mathrm{d} t_{1} \ldots \int \mathrm{d} t_{n} \epsilon^{(n)}\left(t_{1}, \ldots, t_{n}\right) j\left(t-t_{1}\right) \ldots j\left(t-t_{n}\right) \\
& +\ldots
\end{aligned}
$$

Because we expand around the constant solution $x \equiv 0$, the $\epsilon$ kernels depend not on the time $t$ but on the difference $\left(t-t^{*}\right), t^{*}$ being the time when we have stated the initial condition. In this subsection, we calculate the solution for the initial condition $x\left(t^{*}=-\infty\right)=0$ and the $\epsilon$ kernels do not depend on $t$ at all. We have therefore dropped the index $t$ from $\epsilon_{t}^{(n)}$ as it occurs in equation A.4. As is discussed in section A.3, dropping the index is possible only if no spike occurs in the past.

We can easily generalize this formalism to systems of differential equations,

$$
\dot{x_{k}}-f_{k}\left(x_{1}, \ldots, x_{N}\right)=\delta_{k, 1} j(t), \quad k=1, \ldots, N,
$$

and obtain

$$
\begin{aligned}
x_{k}(t)= & \int d t_{1} \epsilon_{k}^{(1)}\left(t_{1}\right) j\left(t-t_{1}\right) \\
& +\frac{1}{2 !} \int \mathrm{d} t_{1} \int \mathrm{d} t_{2} \epsilon_{k}^{(2)}\left(t_{1}, t_{2}\right) j\left(t-t_{1}\right) j\left(t-t_{2}\right)+\cdots
\end{aligned}
$$

As opposed to the lower index $t$ in equation A.4, and the lower index $p$ in equation 2.3, the subscript $k$ in equation A.7 denotes the $k$ th vector component of the system throughout the rest of this appendix.

A.2 Calculation of the Kernels. We want to prove that the kernels $\epsilon_{0}^{(1)}$, $\epsilon_{0}^{(2)}, \ldots$ can be calculated explicitly for fairly arbitrary systems of differential equations. In particular, it is possible to obtain analytic expressions for the kernels in terms of the eigenvalues of the linearization of the $N$-dimensional system of differential equations we start with.

Suppose $f$ in equation A.6 is analytic in the neighborhood of $x=0$ so that we can expand $f$ in a Taylor series around $x=0$. In doing so, we use the Einstein summation convention. We substitute the Taylor series into equation A.6,

$$
\dot{x}_{k}-\left(\frac{\partial f_{k}}{\partial x_{l}} x_{l}+\frac{1}{2 !} \frac{\partial^{2} f_{k}}{\partial x_{l} \partial x_{m}} x_{l} x_{m}+\cdots\right)=\delta_{k, 1} j(t), \quad k=1, \ldots, N,
$$

realize that the derivatives are evaluated at $x=0$, and switch to Fourier transforms,

$$
i \omega x_{k}(\omega)-\left(\frac{\partial f_{k}}{\partial x_{l}} x_{l}(\omega)+\frac{1}{2 !} \frac{\partial^{2} f_{k}}{\partial x_{l} \partial x_{m}} \int d \omega_{1} x_{l}\left(\omega-\omega_{1}\right) x_{m}\left(\omega_{1}\right)+\cdots\right)
$$




$$
=\delta_{k, 1} j(\omega)
$$

The Fourier transform of the Volterra series (see equation A.7) is

$$
\begin{aligned}
x_{k}(\omega)= & \epsilon_{k}^{(1)}(\omega) j(\omega) \\
& +\frac{1}{2 !} \int d \omega_{1} \epsilon_{k}^{(2)}\left(\omega-\omega_{1}, \omega_{1}\right) j\left(\omega-\omega_{1}\right) j\left(\omega_{1}\right)+\cdots
\end{aligned}
$$

We substitute this into equation A.9 and obtain a polynomial (in a convolution algebra) in terms of $j(\omega)$,

$$
\begin{aligned}
0= & {\left[c_{k}+c_{k l}(\omega) \epsilon_{l}^{(1)}(\omega)\right] j(\omega) } \\
& +\int d \omega_{1}\left[\frac{1}{2} c_{k l}(\omega) \epsilon_{l}^{(2)}\left(\omega-\omega_{1}, \omega_{1}\right)+c_{k l m} \epsilon_{l}^{(1)}\left(\omega-\omega_{1}\right) \epsilon_{m}^{(1)}\left(\omega_{1}\right)\right] \\
& \times j\left(\omega-\omega_{1}\right) j\left(\omega_{1}\right) \\
& +\int d \omega_{1} \int d \omega_{2}\left[\frac{1}{6} c_{k l}(\omega) \epsilon_{l}^{(3)}\left(\omega-\omega_{1}-\omega_{2}, \omega_{1}, \omega_{2}\right)+/ \ldots /\right] \\
& \times j\left(\omega-\omega_{1}-\omega_{2}\right) j\left(\omega_{1}\right) j\left(\omega_{2}\right)+\cdots
\end{aligned}
$$

Here we have defined the abbreviations $c_{k}=\delta_{k .1}$,

$$
c_{k l}(\omega)=\frac{\partial f_{k}}{\partial x_{l}}-i \omega \delta_{k . l}
$$

and

$$
c_{k l m}=\frac{1}{2 !} \frac{\partial^{2} f_{k}}{\partial x_{l} \partial x_{m}}, \quad c_{k l m n}=\frac{1}{3 !} \frac{\partial^{3} f_{k}}{\partial x_{l} \partial x_{m} \partial x_{n}}, \quad \ldots
$$

Equation A.11 holds for every function $j$. The coefficients of $j(\omega)$ therefore have to vanish identically, and we are left with a set of linear equations for the kernels, which can be solved consecutively,

$$
\begin{aligned}
c_{k l}(\omega) \epsilon_{l}^{(1)}(\omega) & =-c_{k} \\
c_{k l}\left(\omega_{1}+\omega_{2}\right) \epsilon_{l}^{(2)}\left(\omega_{1}, \omega_{2}\right)= & -2 c_{k l m} \epsilon_{l}^{(1)}\left(\omega_{1}\right) \epsilon_{m}^{(1)}\left(\omega_{2}\right) \\
c_{k l}\left(\omega_{1}+\omega_{2}+\omega_{3}\right) \epsilon_{l}^{(3)}\left(\omega_{1}, \omega_{2}, \omega_{3}\right)= & -6 c_{k l m n} \epsilon_{l}^{(1)}\left(\omega_{1}\right) \epsilon_{m}^{(1)}\left(\omega_{2}\right) \epsilon_{n}^{(1)}\left(\omega_{3}\right) \\
& -3 c_{k l m}\left[\epsilon_{l}^{(1)}\left(\omega_{1}\right) \epsilon_{m}^{(2)}\left(\omega_{2}, \omega_{3}\right)\right. \\
& \left.+\epsilon_{m}^{(1)}\left(\omega_{2}\right) \epsilon_{l}^{(2)}\left(\omega_{1}, \omega_{3}\right)\right]
\end{aligned}
$$


Note that we have to invert only one matrix, $c_{k l}(\omega)$, in order to solve equation A.14 for all kernels. The inverse of $c_{k l}(\omega)$ is

$$
c_{m n}^{-1}(\omega)=\frac{1}{\operatorname{det} c_{k !}(\omega)} C_{n m}(\omega)
$$

where $C_{n m}(\omega)$ is the adjunct matrix of $c_{k l}(\omega)$ defined in equation A.12.

Solving equation A.14, we obtain for $\epsilon_{k}^{(n)}\left(\omega_{1}, \ldots, \omega_{n}\right)$ an expression with a denominator that is a product of the characteristic polynomial $p(\omega):=$ $\operatorname{det} c_{k l}(\omega)$, evaluated at different frequencies $\omega$. For instance, solving equation A.14 for $\epsilon_{k}^{(2)}\left(\omega_{1}, \omega_{2}\right)$ yields a denominator $p\left(\omega_{1}\right) p\left(\omega_{2}\right) p\left(\omega_{1}+\omega_{2}\right)$, and the denominator in $\epsilon_{k}^{(3)}\left(\omega_{1}, \omega_{2}, \omega_{3}\right)$ is $p\left(\omega_{1}\right) p\left(\omega_{2}\right) p\left(\omega_{3}\right) p\left(\omega_{1}+\omega_{3}\right) p\left(\omega_{2}+\right.$ $\left.\omega_{3}\right) p\left(\omega_{1}+\omega_{2}+\omega_{3}\right)$, and so forth. If we know the roots of $p(\omega)$, that is, the eigenvalues of the system of differential equations, we can factorize the denominator and easily determine the residues of $\epsilon_{k}^{(n)}\left(\omega_{1}, \ldots, \omega_{n}\right)$ and thus derive an analytic expression for the inverse Fourier transform of $\epsilon_{k}^{(n)}\left(\omega_{1}, \ldots, \omega_{n}\right)$,

$$
\epsilon_{k}^{(n)}\left(t_{1}, \ldots, t_{n}\right)=\theta\left(t_{1}, \ldots, t_{n}\right) \sum_{l} a_{k l} \exp \left[v_{k l 1} t_{1}+\cdots+v_{k l n} t_{n}\right]
$$

The coefficients $\nu_{k l m} \in \mathbb{C}$ are sums and differences of the $N$ eigenvalues $\lambda_{k}$ of the $\mathrm{N}$-dimensional system of differential equations,

$$
v_{k l m}=\varsigma_{k l m 1} \lambda_{1}+\cdots+\varsigma_{k l m N} \lambda_{N}, \quad \varsigma \in\{-1,0,1\}
$$

The computational difficulties are reduced to the calculation of the $N$ roots of a polynomial of degree $N$ where $N$ is the system dimension; see equation A.6.

A.3 Limitations. In most cases, the function $F$ is not an entire function; in other words, the series expansion around the constant solution $x \equiv 0$ does not converge for all perturbations $j$ (Thomas, 1996). For the Hodgkin-Huxley equations, we have found numerically that the truncated series expansion gives a fine approximation to the true solution only as long as the input current keeps well below the spiking threshold. This is not too surprising, since the solution of Hodgkin-Huxley equations exhibits an apparent discontinuity at spiking threshold. Consider a family of input functions given by

$$
j_{\tau}: t \mapsto j_{0} \theta(t-\tau) \theta(t+\tau)
$$

These are square pulses with height $j_{0}$ and duration $2 \tau$. There is a threshold $j_{9}(\tau)$ and a small constant $\delta$ with $\delta / j_{9}(\tau) \ll 1$ so that for $j_{0}<j_{9}(\tau)-\delta$ 
no spike occurs, whereas for $j_{0}>j_{\vartheta}(\tau)+\delta$ at least one spike is triggered within a given compact interval (see Figure 2). Thus, the two-norm of the derivative, $\left\|F^{\prime}\left[j_{\tau}\right]\right\|_{2} \approx\left\|F\left[j_{\tau}+\delta\right]-F\left[j_{\tau}-\delta\right]\right\|_{2} / 2 \delta$, takes large values as $j_{0}$ approaches the value $j_{\vartheta}(\tau)$, and we do not expect the series expansion to converge beyond that point.

In the body of this article, we introduced the response function $\eta$ in order to address this problem. The key advantage of $\eta_{1}$ and the "improved" kernels $\epsilon_{1}^{(1)}, \epsilon_{1}^{(2)}, \ldots$ is that we no longer expand around the constant solution $x \equiv 0$ but around a solution of the Hodgkin-Huxley equations that contains a single spike at $t=t^{f}$. In the context of equation A.5, we have argued that the $\epsilon$ kernels do not depend on the absolute time. However, since the new zero-order approximation $u(t)=\eta_{1}\left(t-t^{f}\right)$ contains a spike, homogeneity of time is destroyed and the improved $\epsilon$ kernels depend on $\left(t-t^{f}\right)$ where $t^{f}$ is the latest firing time. Unfortunately, there is no analytic solution for the Hodgkin-Huxley equations with spikes. We therefore have to, and did, resort to numerical methods in order to determine the kernel $\epsilon_{1}^{(n)}$ in the neighborhood of a spike.

In order to construct approximations of solutions with more than one spike, we have to concatenate these single-spike approximations. We can then exploit the fact that the truncated series expansion exhibits rather prominent peaks in the membrane potential at positions where the HodgkinHuxley equations would produce a spike. The task is to decide from the truncated series expansion which of the peaks in the approximated membrane potential belong to a spike and which do not. In the main body of the article, we investigated how well this can be done by using a threshold criterion.

\section{Acknowledgments}

This work was supported by the Deutsche Forschungsgemeinschaft (DFG) under grant numbers He 1729/2-2 and 8-1.

\section{References}

Abbott, L. F., \& Kepler, T. B. (1990). Model neurons: From Hodgkin-Huxley to Hopfield. In L. Garrido (Ed.), Statistical Mechanics of Neural Networks. Berlin: Springer-Verlag.

Abbott, L. F., \& Vreeswijk, C. van. (1993). Asynchronous states in a network of pulse-coupled oscillators. Phys. Rev. E 48:1483-1490.

Av-Ron, E., Parnas, H., \& Segel, L. A. (1991). A minimal biophysical model for an excitable and oscillatory neuron. Biol. Cubern. 65:487-500.

Cole, K. S., Guttman, R., \& Bezanilla, F. (1970). Nerve membrane excitation without threshold. Proc. Natl Acad. Sci. 65(4):884-891.

Cronin, J. (1987). Mathematical aspects of Hodgkin-Huxley theory. Cambridge: Cambridge University Press. 
Dieudonné, J. (1968). Foundations of modern analysis. New York: Academic Press.

Dunford, N., \& Schwartz, J. T. (1958). Linear operators, Part I: General theory. New York: Wiley.

Ekeberg, O., Wallen, P., Lansner, A., Traven, H., Brodin, L., \& Grillner, S. (1991). A computer based model for realistic simulations of neural networks. Biol. Cybern. 65:81-90.

Feller, W. (1970). An Introduction to Probability Theory and Its Applications (3rd ed., Vol. 1). New York: Wiley.

FitzHugh, R. (1961). Impulses and physiological states in models of nerve membrane. Biophys. J. 1:445-466.

Gerstner, W. (1991). Associative memory in a network of "biological" neurons. In R. P. Lippmann, J. E. Moody, \& D. S. Touretzky (Eds.), Advances in Neural Information Processing Systems 3 (pp. 84-90). San Mateo, CA: Morgan Kaufmann.

Gerstner, W. (1995). Time structure of the activity in neural network models. Bhus. Rev. E 51:738-758.

Gerstner, W., \& Hemmen, J. L. van. (1992). Associative memory in a network of "spiking" neurons. Network 3:139-164.

Gerstner, W., \& Hemmen, J. L. van. (1993). Coherence and incoherence in a globally coupled ensemble of pulse emitting units. Phus. Rev. Lett. 71(3):312315.

Gerstner, W., Hemmen, J. L. van, \& Cowan, J. D. (1996). What matters in neuronal locking? Neural Comp. 8:1689-1712.

Hille, E., \& Phillips, R. S. (1957). Functional analysis and semi-groups. Providence, RI: American Mathematical Society.

Hodgkin, A. L., \& Huxley, A. F. (1952). A quantitative description of ion currents and its applications to conduction and excitation in nerve membranes. J. Physiol. (London) 117:500-544.

Hopfield, J. J., \& Herz, A. V. M. (1995). Rapid local synchronization of action potentials: Towards computation with coupled integrate-and-fire networks. Proc. Natl. Acad. Sci. USA 92:6655.

Joeken, S., \& Schwegler, H. (1995). Predicting spike train responses of neuron models. In M. Verleysen (Ed.), Proc. 3rd European Symposium on Artificial Neural Networks (pp. 93-98). Brussels: D facto.

Kepler, Thomas B., Abbott, L. F., \& Marder, E. (1992). Reduction of conductancebased neuron models. Biol. Cubern. 66:381-387.

Kernell, D., \& Sjöholm, H. (1973). Repetitive impulse firing: Comparison between neuron models based on voltage clamp equations and spinal motoneurons. Acta Physiol. Scand. 87:40-56.

Koch, C., Bernander, Ö., \& Douglas, R. J. (1995). Do neurons have a voltage or a current threshold for action potential initiation? J. Comp. Neurosci. 2:63-82.

Kolmogorov, A. N., \& Fomin, S. V. (1975). Reelle Funktionen und Funktionalanalysis. Berlin: VEB Deutscher Verlag der Wissenschaften.

Korenberg, M. J. (1989). A robust orthogonal algorithm for system identification and time-series analysis. Biol. Cybern. 60:267-276.

Lapicque, L. (1907). Recherches quantitatives sur l'excitation électrique des nerfs traitée comme une polarisation. J. Physiol. Pathol. Gen. 9:620-635. 
Mainen, Z. F., \& Sejnowski, T. J. (1995). Reliability of spike timing in neocortical neurons. Science 268:1503-1506.

Mauro, A., Conti, F., Dodge, F., \& Schor, R. (1970). Subthreshold behavior and phenomenological impedance of the giant squid axon. I. Gen. Physiol. 55:497523.

Palm, G. (1978). On representation and approximation of nonlinear systems. Biol. Cubernetics 31:119-124.

Palm, G., \& Poggio, T. (1977). The Volterra representation and the Wiener expansion: Validity and pitfalls. SIAM J. Appl. Math. 33(2):195-216.

Palm, G., \& Poggio, T. (1978). Stochastic identification methods for nonlinear systems: An extension of the Wiener theory. SIAM J. Appl. Math. 34(3):524534.

Prohorov, Yu. V., \& Rozanov, Yu. A. (1969). Probability. Berlin: Springer-Verlag.

Rinzel, J. (1985). Excitation dynamics: Insights from simplified membrane models. Federation Proc. 44:2944-2946.

Rinzel, J., \& Ermentrout, G. B. (1989). Analysis of neuronal excitability and oscillations. In C. Koch \& I. Segev (Eds.), Methods in Neuronal Modeling. Cambridge, MA: MIT Press.

Thomas, E. G. F. (1996). Q-analytic solution operators for non-linear differential equations (Preprint). Department of Mathematics, University of Groningen.

Traub, R. D., Wong, R. K. S., Miles, R., \& Michelson, H. (1991). A model of a CA3 hippocampal pyramidal neuron incorporating voltage-clamp data on intrinsic conductances. J. Neurophysiol. 66:635-650.

Tsodyks, M., Mitkov, I., \& Sompolinsky, H. (1993). Patterns of synchrony in inhomogeneous networks of oscillators with pulse interaction. Phys. Rev. Lett. 71:1281-1283.

Usher, M., Schuster, H. G., \& Niebur, E. (1993). Dynamics of populations of integrate-and-fire neurons, partial synchronization and memory. Neural Computation 5:570-586.

Volterra, V. (1959). Theory of Functionals and of Integral and Integro-Differential Equations. New York: Dover.

Wiener, N. (1958). Nonlinear Problems in Random Theory. Cambridge, MA: MIT Press.

Wilson, M. A., Bhalla, U. S., Uhley, J. D., \& Bower, J. M. (1989). Genesis: A system for simulating neural networks. In D. Touretzky (Ed.), Advances in Neural Information Processing Systems (pp. 485-492). San Mateo, CA: Morgan Kaufmann.

Yamada, W. M., Koch, C., \& Adams, P. R. (1989). Multiple channels and calcium dynamics. In C. Koch \& I. Segev (Eds.), Methods in Neuronal Modeling: From Synapses to Networks. Cambridge, MA: MIT Press.

Received April 17, 1996; accepted October 28, 1996. 


\section{This article has been cited by:}

2. Anthony R. Kellems, Saifon Chaturantabut, Danny C. Sorensen, Steven J. Cox. 2010. Morphologically accurate reduced order modeling of spiking neurons. Journal of Computational Neuroscience . [CrossRef]

3. Enrico Bibbona, Roberta Sirovich. 2010. Estimating input parameters from intracellular recordings in the Feller neuronal model. Physical Review E 81:3. . [CrossRef]

4. Toshiaki Omori, Toru Aonishi, Masato Okada. 2010. Switch of encoding characteristics in single neurons by subthreshold and suprathreshold stimuli. Physical Review E 81:2. . [CrossRef]

5. Louis Tao, Andrew T. Sornborger. 2010. Dimensionally-reduced visual cortical network model predicts network response and connects system- and cellular-level descriptions. Journal of Computational Neuroscience 28:1, 91-106. [CrossRef]

6. Anthony R. Kellems, Derrick Roos, Nan Xiao, Steven J. Cox. 2009. Low-dimensional, morphologically accurate models of subthreshold membrane potential. Journal of Computational Neuroscience 27:2, 161-176. [CrossRef]

7. Eugenio Urdapilleta, Inés Samengo. 2009. Quasistatic approximation of the interspike interval distribution of neurons driven by time-dependent inputs. Physical Review E 80:1. . [CrossRef]

8. Benjamin Lindner. 2009. Some unsolved problems relating to noise in biological systems. Journal of Statistical Mechanics: Theory and Experiment 2009:01, P01008. [CrossRef]

9. Michael London, Matthew E. Larkum, Michael Häusser. 2008. Predicting the synaptic information efficacy in cortical layer 5 pyramidal neurons using a minimal integrate-and-fire model. Biological Cybernetics 99:4-5, 393-401. [CrossRef]

10. Renaud Jolivet, Felix Schürmann, Thomas K. Berger, Richard Naud, Wulfram Gerstner, Arnd Roth. 2008. The quantitative single-neuron modeling competition. Biological Cybernetics 99:4-5, 417-426. [CrossRef]

11. Rama Natarajan, Quentin J. M. Huys, Peter Dayan, Richard S. Zemel. 2008. Encoding and Decoding Spikes for Dynamic StimuliEncoding and Decoding Spikes for Dynamic Stimuli. Neural Computation 20:9, 2325-2360. [Abstract] [PDF] [PDF Plus]

12. P. Rowcliffe, Jianfeng Feng. 2008. Training Spiking Neuronal Networks With Applications in Engineering Tasks. IEEE Transactions on Neural Networks 19:9, 1626-1640. [CrossRef]

13. Enrico Bibbona, Petr Lansky, Laura Sacerdote, Roberta Sirovich. 2008. Errors in estimation of the input signal for integrate-and-fire neuronal models. Physical Review E 78:1. . [CrossRef] 
14. Lawrence Sirovich. 2008. Populations of Tightly Coupled Neurons: The RGC/LGN SystemPopulations of Tightly Coupled Neurons: The RGC/LGN System. Neural Computation 20:5, 1179-1210. [Abstract] [PDF] [PDF Plus]

15. Alexander Casti, Fernand Hayot, Youping Xiao, Ehud Kaplan. 2008. A simple model of retina-LGN transmission. Journal of Computational Neuroscience 24:2, 235-252. [CrossRef]

16. Sungho Hong, Blaise Agüera y Arcas, Adrienne L. Fairhall. 2007. Single Neuron Computation: From Dynamical System to Feature DetectorSingle Neuron Computation: From Dynamical System to Feature Detector. Neural Computation 19:12, 3133-3172. [Abstract] [PDF] [PDF Plus]

17. Tobias Prager, Hans-Philipp Lerch, Lutz Schimansky-Geier, Eckehard Schöll. 2007. Increase of coherence in excitable systems by delayed feedback. Journal of Physics A: Mathematical and Theoretical 40:36, 11045-11055. [CrossRef]

18. Ryota Kobayashi, Shigeru Shinomoto. 2007. State space method for predicting the spike times of a neuron. Physical Review E 75:1. . [CrossRef]

19. Renaud Jolivet, Alexander Rauch, Hans-Rudolf Lüscher, Wulfram Gerstner. 2006. Predicting spike timing of neocortical pyramidal neurons by simple threshold models. Journal of Computational Neuroscience 21:1, 35-49. [CrossRef]

20. A. N. Burkitt. 2006. A Review of the Integrate-and-fire Neuron Model: I. Homogeneous Synaptic Input. Biological Cybernetics 95:1, 1-19. [CrossRef]

21. Susanne Ditlevsen, Petr Lansky. 2006. Estimation of the input parameters in the Feller neuronal model. Physical Review E 73:6. . [CrossRef]

22. Yuval Aviel, Wulfram Gerstner. 2006. From spiking neurons to rate models: A cascade model as an approximation to spiking neuron models with refractoriness. Physical Review E 73:5. . [CrossRef]

23. P. Rowcliffe, J. Feng, H. Buxton. 2006. Spiking Perceptrons. IEEE Transactions on Neural Networks 17:3, 803-807. [CrossRef]

24. Christo Panchev, Stefan Wermter. 2006. Temporal sequence detection with spiking neurons: towards recognizing robot language instructions. Connection Science 18:1, 1-22. [CrossRef]

25.Xuedong Zhang, Laurel H. Carney . 2005. Response Properties of an Integrate-and-Fire Model That Receives Subthreshold InputsResponse Properties of an Integrate-and-Fire Model That Receives Subthreshold Inputs. Neural Computation 17:12, 2571-2601. [Abstract] [PDF] [PDF Plus]

26. A. Tonnelier . 2005. Categorization of Neural Excitability Using Threshold ModelsCategorization of Neural Excitability Using Threshold Models. Neural Computation 17:7, 1447-1455. [Abstract] [PDF] [PDF Plus]

27. S. Roy, V. Beiu. 2005. Majority Multiplexing-Economical Redundant Fault-Tolerant Designs for Nanoarchitectures. IEEE Transactions On Nanotechnology 4:4, 441-451. [CrossRef] 
28. Christian Leibold, J. van Hemmen. 2005. Spiking Neurons Learning Phase Delays: How Mammals May Develop Auditory Time-Difference Sensitivity. Physical Review Letters 94:16. . [CrossRef]

29. Robert Clewley, Horacio G. Rotstein, Nancy Kopell. 2005. A Computational Tool for the Reduction of Nonlinear ODE Systems Possessing Multiple Scales. Multiscale Modeling \& Simulation 4:3, 732. [CrossRef]

30. V. Beiu, J.M. Quintana, M.J. Avedillo. 2003. VLSI implementations of threshold logic- a comprehensive survey. IEEE Transactions on Neural Networks 14:5, 1217-1243. [CrossRef]

31. Blaise Agüera y Arcas, Adrienne L. Fairhall . 2003. What Causes a Neuron to Spike?What Causes a Neuron to Spike?. Neural Computation 15:8, 1789-1807. [Abstract] [PDF] [PDF Plus]

32. Blaise Agüera y Arcas, Adrienne L. Fairhall, William Bialek . 2003. Computation in a Single Neuron: Hodgkin and Huxley RevisitedComputation in a Single Neuron: Hodgkin and Huxley Revisited. Neural Computation 15:8, 1715-1749. [Abstract] [PDF] [PDF Plus]

33. Jianfeng Feng, Henry C. Tuckwell. 2003. Optimal Control of Neuronal Activity. Physical Review Letters 91:1. . [CrossRef]

34. Arnaud Tonnelier, Wulfram Gerstner. 2003. Piecewise linear differential equations and integrate-and-fire neurons: Insights from two-dimensional membrane models. Physical Review E 67:2. . [CrossRef]

35. Werner M. Kistler, Wulfram Gerstner . 2002. Stable Propagation of Activity Pulses in Populations of Spiking NeuronsStable Propagation of Activity Pulses in Populations of Spiking Neurons. Neural Computation 14:5, 987-997. [Abstract] [PDF] [PDF Plus]

36. A. N. Burkitt , G. M. Clark . 2001. Synchronization of the Neural Response to Noisy Periodic Synaptic InputSynchronization of the Neural Response to Noisy Periodic Synaptic Input. Neural Computation 13:12, 2639-2672. [Abstract] [PDF] [PDF Plus]

37. Ken-ichi Amemori , Shin Ishii . 2001. Gaussian Process Approach to Spiking Neurons for Inhomogeneous Poisson InputsGaussian Process Approach to Spiking Neurons for Inhomogeneous Poisson Inputs. Neural Computation 13:12, 2763-2797. [Abstract] [PDF] [PDF Plus]

38. J. Eggert , J. L. van Hemmen . 2001. Modeling Neuronal Assemblies: Theory and ImplementationModeling Neuronal Assemblies: Theory and Implementation. Neural Computation 13:9, 1923-1974. [Abstract] [PDF] [PDF Plus]

39. M. C. W. van Rossum . 2001. A Novel Spike DistanceA Novel Spike Distance. Neural Computation 13:4, 751-763. [Abstract] [PDF] [PDF Plus]

40. Ulrich Hillenbrand, J. Leo van Hemmen . 2001. Does Corticothalamic Feedback Control Cortical Velocity Tuning?Does Corticothalamic Feedback Control Cortical 
Velocity Tuning?. Neural Computation 13:2, 327-355. [Abstract] [PDF] [PDF Plus]

41. H. Torikai, T. Saito. 2001. Resonance phenomenon of interspike intervals from a spiking oscillator with two periodic inputs. IEEE Transactions on Circuits and Systems I Fundamental Theory and Applications 48:10, 1198. [CrossRef]

42. Werner Kistler. 2000. Stability properties of solitary waves and periodic wave trains in a two-dimensional network of spiking neurons. Physical Review E 62:6, 8834-8837. [CrossRef]

43. A. N. Burkitt , G. M. Clark . 2000. Calculation of Interspike Intervals for Integrate-and-Fire Neurons with Poisson Distribution of Synaptic InputsCalculation of Interspike Intervals for Integrate-and-Fire Neurons with Poisson Distribution of Synaptic Inputs. Neural Computation 12:8, 1789-1820. [Abstract] [PDF] [PDF Plus]

44. Werner M. Kistler , J. Leo van Hemmen . 2000. Modeling Synaptic Plasticity in Conjunction with the Timing of Pre- and Postsynaptic Action PotentialsModeling Synaptic Plasticity in Conjunction with the Timing of Pre- and Postsynaptic Action Potentials. Neural Computation 12:2, 385-405. [Abstract] [PDF] [PDF Plus]

45. Paul C. Bressloff , S. Coombes . 2000. Dynamics of Strongly Coupled Spiking NeuronsDynamics of Strongly Coupled Spiking Neurons. Neural Computation 12:1, 91-129. [Abstract] [PDF] [PDF Plus]

46. Wulfram Gerstner . 2000. Population Dynamics of Spiking Neurons: Fast Transients, Asynchronous States, and LockingPopulation Dynamics of Spiking Neurons: Fast Transients, Asynchronous States, and Locking. Neural Computation 12:1, 43-89. [Abstract] [PDF] [PDF Plus]

47. P. C. Bressloff, S. Coombes. 2000. Solitary Waves in a Model of Dendritic Cable with Active Spines. SIAM Journal on Applied Mathematics 61:2, 432. [CrossRef]

48. Erik G. F. Thomas, J. Leo van Hemmen, Werner M. Kistler. 2000. Calculation of Volterra Kernels for Solutions of Nonlinear Differential Equations. SIAM Journal on Applied Mathematics 61:1, 1. [CrossRef]

49. Werner M. Kistler, J. Leo van Hemmen . 1999. Short-Term Synaptic Plasticity and Network BehaviorShort-Term Synaptic Plasticity and Network Behavior. Neural Computation 11:7, 1579-1594. [Abstract] [PDF] [PDF Plus]

50. Hans Plesser, Theo Geisel. 1999. Markov analysis of stochastic resonance in a periodically driven integrate-and-fire neuron. Physical Review E 59:6, 7008-7017. [CrossRef]

51. I.C. Bruce, M.W. White, L.S. Irlicht, S.J. O'Leary, S. Dynes, E. Javel, G.M. Clark. 1999. A stochastic model of the electrically stimulated auditory nerve: single-pulse response. IEEE Transactions on Biomedical Engineering 46:6, 617-629. [CrossRef]

52. A. N. Burkitt , G. M. Clark . 1999. Analysis of Integrate-and-Fire Neurons: Synchronization of Synaptic Input and Spike OutputAnalysis of Integrate-and-Fire 
Neurons: Synchronization of Synaptic Input and Spike Output. Neural Computation 11:4, 871-901. [Abstract] [PDF] [PDF Plus]

53. Richard Kempter, Wulfram Gerstner, J. Leo van Hemmen, Hermann Wagner . 1998. Extracting Oscillations: Neuronal Coincidence Detection with Noisy Periodic Spike InputExtracting Oscillations: Neuronal Coincidence Detection with Noisy Periodic Spike Input. Neural Computation 10:8, 1987-2017. [Abstract] [PDF] [PDF Plus] 